\title{
NHC Core Phosphonium Ylide-Based Palladium(II) Pincer Complexes: The Second Ylide Extremity Makes the Difference
}

\author{
Rachid Taakili, Cécile Barthes, Amel Goëffon, Christine Lepetit, \\ Carine Duhayon, Dmitry A. Valyaev, Yves Canac*
}

LCC-CNRS, Université de Toulouse, CNRS, 205 route de Narbonne 31077 Toulouse cedex 4, France.

\section{Supporting Information}

\section{Table of contents}

- NMR spectra of [2](OTf) 3 , Fig. S1-S3

- NMR spectra of [3](OTf), Fig. S4-S6

- NMR spectra of [4](OTf), Fig. S7-S9

- NMR spectra of [5a](OTf), Fig. S10-S12

- NMR spectra of [5b](OTf), Fig. S13-S15

- IR spectrum of [5b](OTf), Fig. S16

- NMR spectra of [7]Br2, Fig. S17-S19

- NMR spectra of [7](OTf) 2 , Fig. S20-S22

- NMR spectra of [8](OTf), Fig. S23-S25

- NMR spectra of [9](OTf)2, Fig. S26-S28

- NMR spectra of 10, Fig. S29-S31

- NMR spectra of [11a](OTf), Fig. S32-S34

- NMR spectra of [11b](OTf), Fig. S35-S37

- IR spectrum of $[\mathbf{1 1 b}](\mathrm{OTf})$, Fig. S38

- Crystallographic table for [4](OTf), [7] $\mathrm{Br}_{2},[8](\mathrm{OTf}),[9](\mathrm{OTf})_{2}$, and 10, Table S1

- Optimized structures of Pd complex $\mathbf{5}^{\mathbf{2}}$, Fig. S39

- Optimized structures of Pd complex $\mathbf{1 1}^{+}$, Fig. S40

- Optimized structures of Pd complex $\mathbf{1 2}^{\mathbf{2}}$, Fig. S41

- Optimized structures of Pd complex 13 ${ }^{+}$, Fig. S42 
Fig. S1. ${ }^{31} \mathbf{P}\left\{{ }^{1} \mathbf{H}\right\}$ NMR spectrum of $[2](O T f)_{3}\left(\right.$ in $\left.\mathrm{CD}_{3} \mathrm{CN}\right)$

$\begin{array}{llllllllllllllllllllllllllllllllllllllll}100 & 95 & 90 & 85 & 80 & 75 & 70 & 65 & 60 & 55 & 50 & 45 & 40 & 35 & 30 & 25 & 20 & 15 & 10 & 5 & 0 & -5 & -10 & -15 & -20 & -25 & -30 & -35 & -40 & -45 & -5\end{array}$

Fig. S2. ${ }^{1} \mathrm{H}$ NMR spectrum of $[2](\text { OTf })_{3}\left(\right.$ in $\left.\mathrm{CD}_{3} \mathrm{CN}\right)$

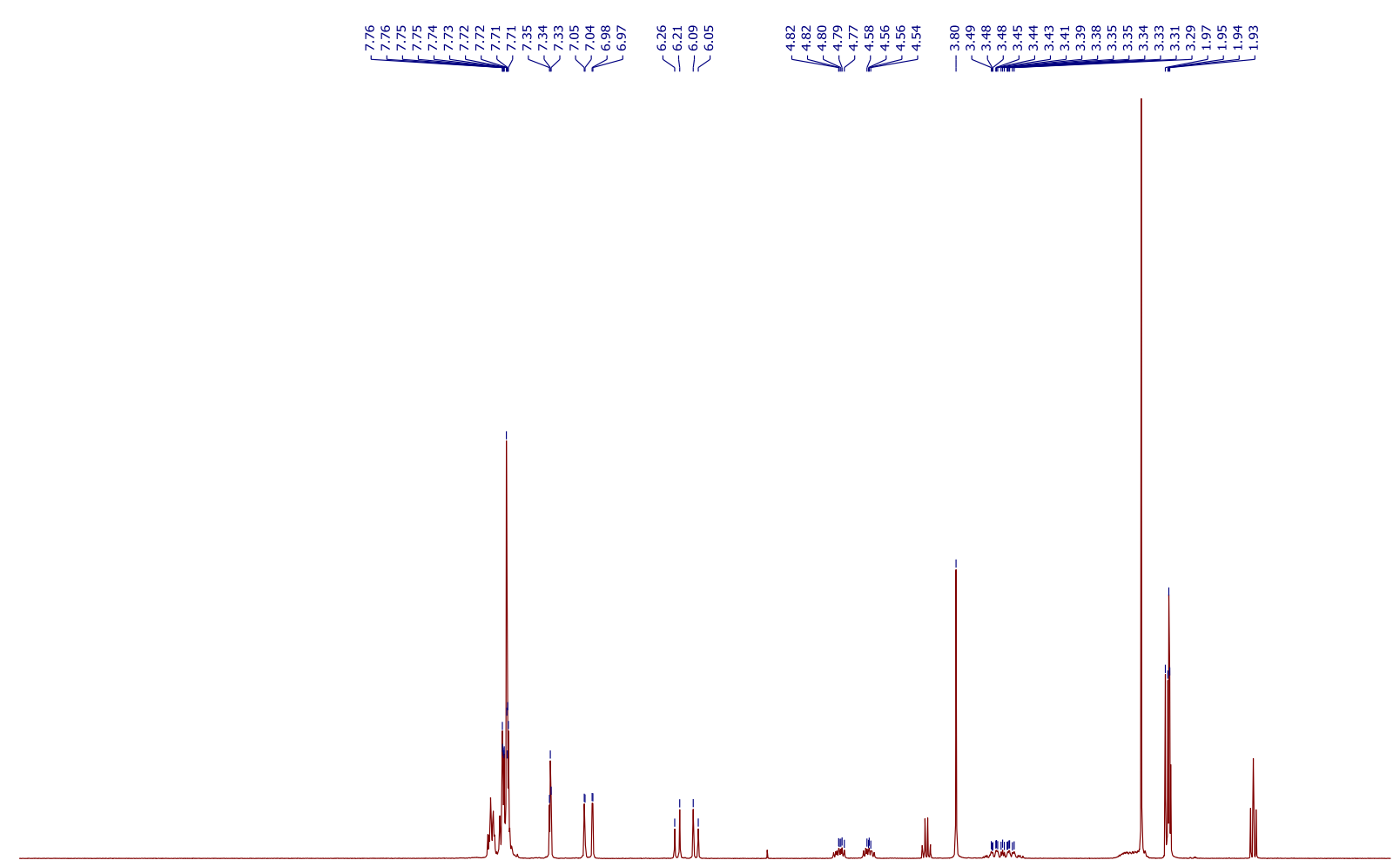

$\begin{array}{lllllllllllllllllllllll}11.5 & 11.0 & 10.5 & 10.0 & 9.5 & 9.0 & 8.5 & 8.0 & 7.5 & 7.0 & 6.5 & \begin{array}{c}6.0 \\ \mathrm{f}(\mathrm{ppm})\end{array} & 5.5 & 5.0 & 4.5 & 4.0 & 3.5 & 3.0 & 2.5 & 2.0 & 1.5 & 1.0 & 0.5\end{array}$ 
Fig. S3. ${ }^{13} \mathbf{C}\left\{{ }^{1} \mathbf{H}\right\}$ NMR spectrum of $[2](\text { OTf })_{3}\left(\right.$ in $\left.\mathrm{CD}_{3} \mathrm{CN}\right)$
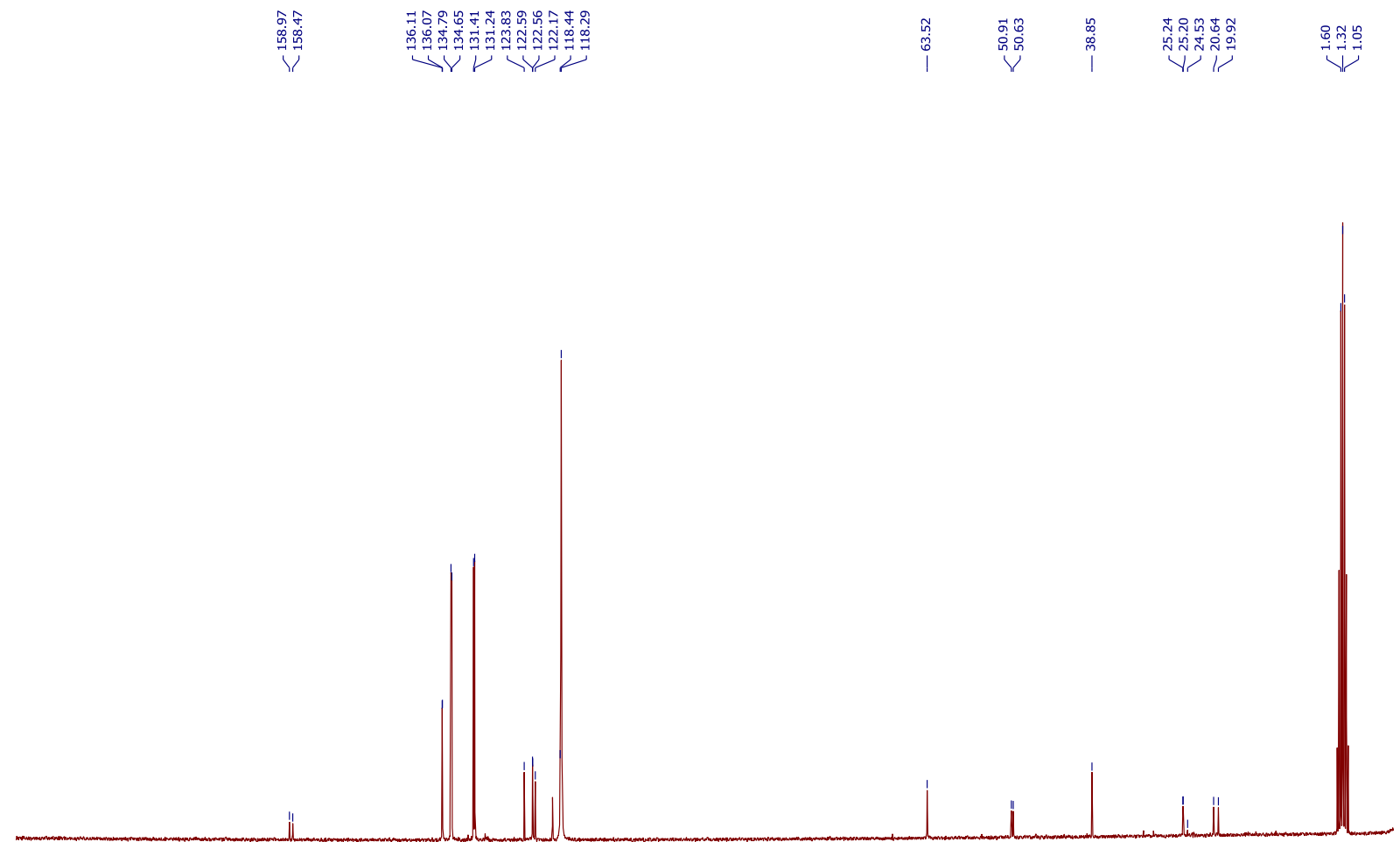

$\begin{array}{lllllllllll}190 & 180 & 170 & 160 & 150 & 140 & 130 & 120 & 110 & \underset{\mathrm{f} 1(\mathrm{ppm})}{100} & 80\end{array}$

Fig. S4. ${ }^{31} \mathbf{P}\left\{{ }^{1} \mathbf{H}\right\}$ NMR spectrum of [3](OTf) (in $\mathrm{CD}_{3} \mathrm{CN}$ ) 
Fig. S5. ${ }^{1} \mathrm{H}$ NMR spectrum of [3](OTf) (in $\mathrm{CD}_{3} \mathrm{CN}$ )

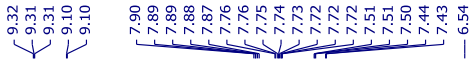

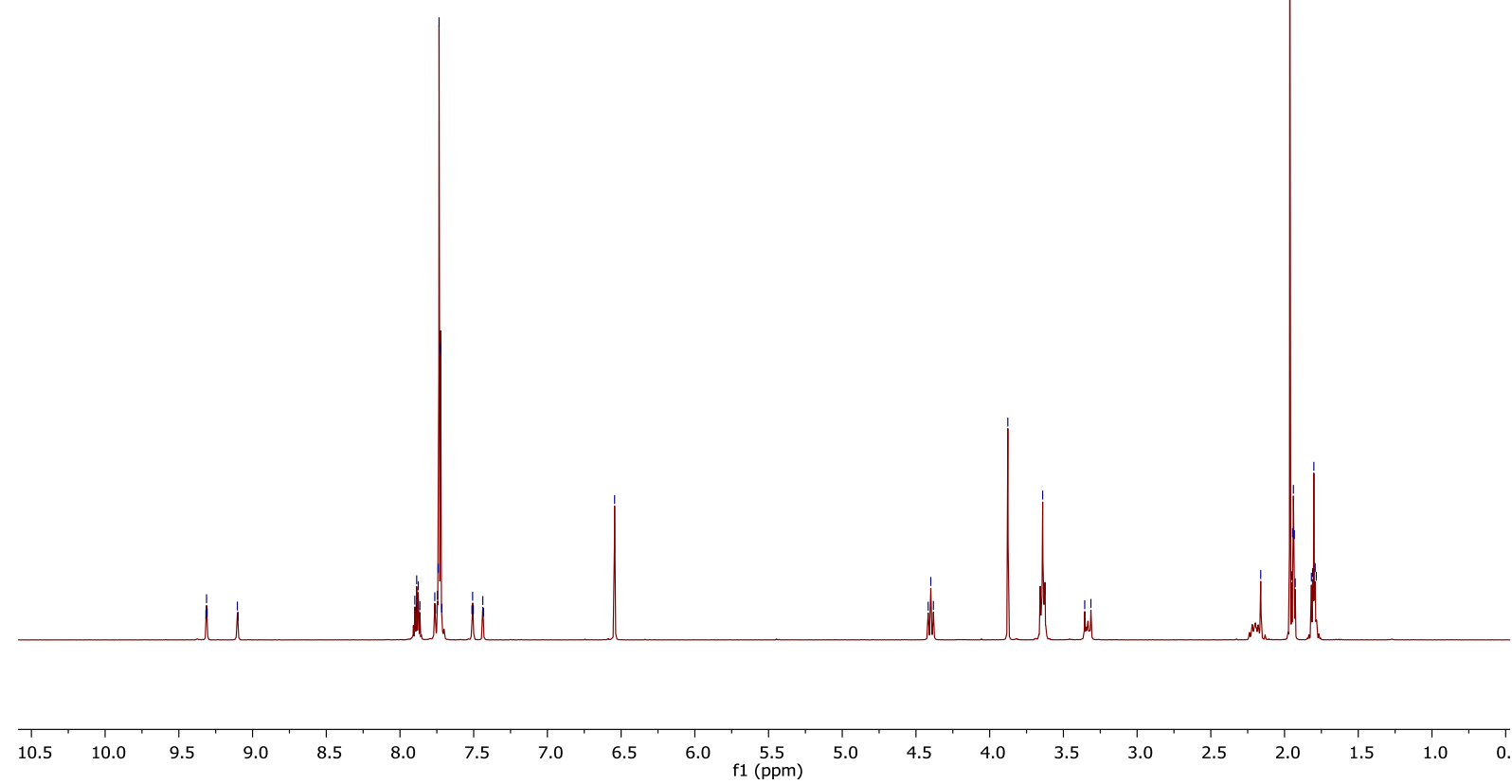

Fig. S6. ${ }^{13} \mathbf{C}\left\{{ }^{1} \mathbf{H}\right\}$ NMR spectrum of [3](OTf) (in $\mathrm{CD}_{3} \mathrm{CN}$ )

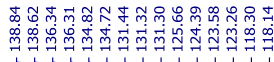

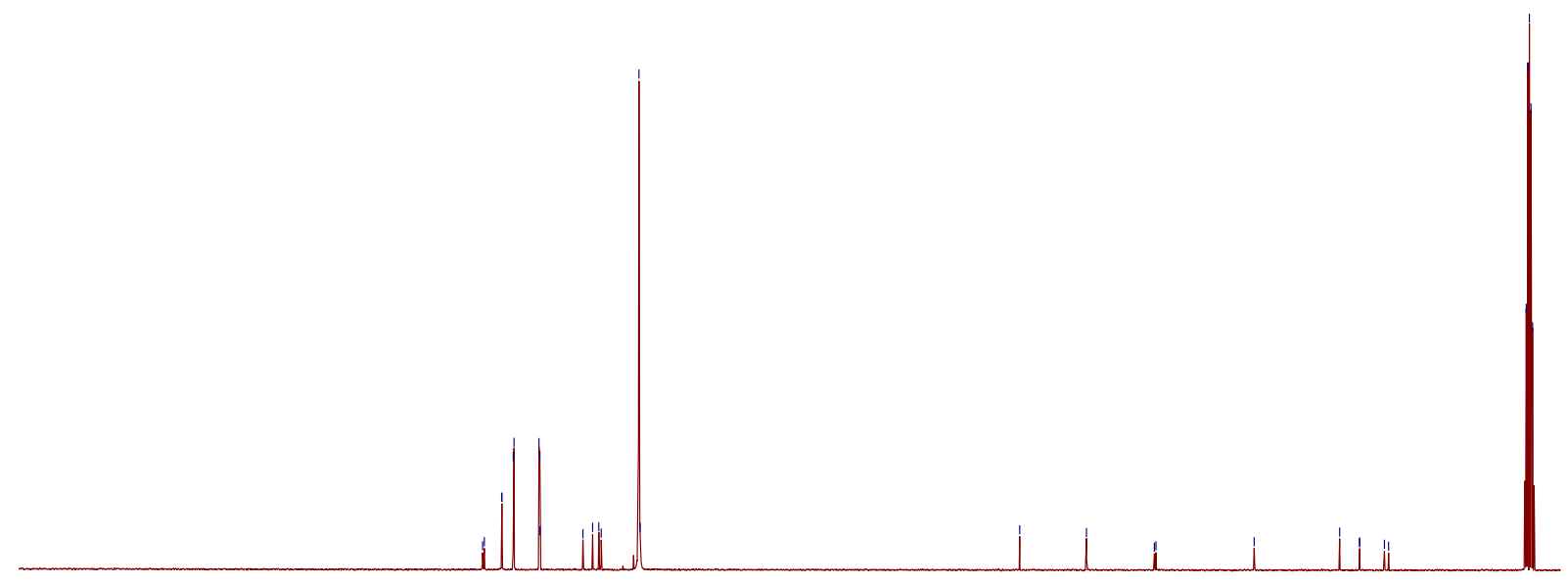

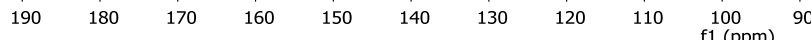

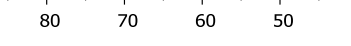

(3)

$10 \quad 0$ 
Fig. S7. ${ }^{31} \mathbf{P}\left\{{ }^{1} \mathbf{H}\right\}$ NMR spectrum of $[4](\mathbf{O T f})\left(\right.$ in $\left.\mathrm{CD}_{3} \mathrm{CN}\right)$

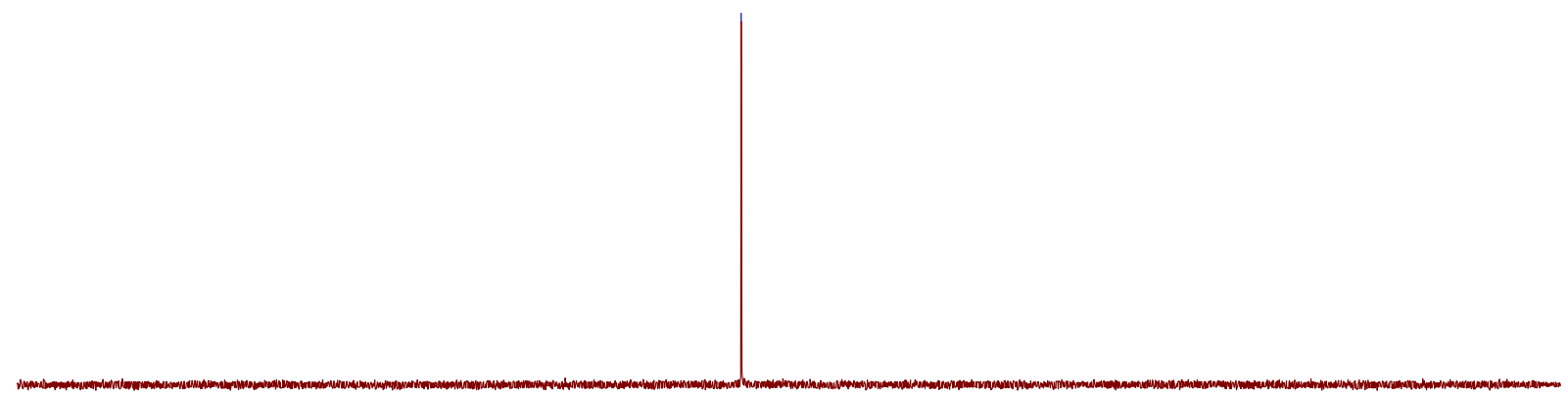

$\begin{array}{llllllllllllllllllllllllllllllll}100 & 95 & 90 & 85 & 80 & 75 & 70 & 65 & 60 & 55 & 50 & 45 & 40 & 35 & 30 & 25 & 20 & 15 & 10 & 5 & 0 & -5 & -10 & -15 & -20 & -25 & -30 & -35 & -40 & -45 & -5\end{array}$

Fig. S8. ${ }^{1} \mathrm{H}$ NMR spectrum of $[4](\mathrm{OTf})\left(\right.$ in $\left.\mathrm{CD}_{3} \mathrm{CN}\right)$

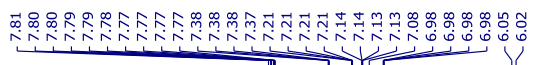

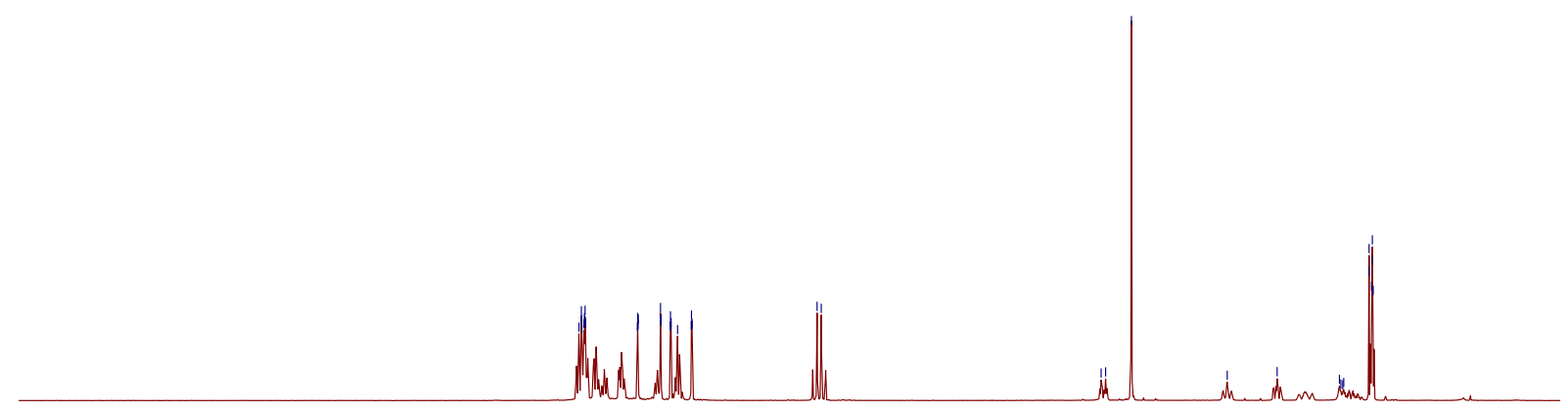

$\begin{array}{llllllllllllllllllllll}11.5 & 11.0 & 10.5 & 10.0 & 9.5 & 9.0 & 8.5 & 8.0 & 7.5 & 7.0 & \underset{f}{6}(\mathrm{ppm}) & 6.0 & 5.5 & 5.0 & 4.5 & 4.0 & 3.5 & 3.0 & 2.5 & 2.0 & 1.5 & 1.0\end{array}$ 
Fig. S9. ${ }^{13} \mathbf{C}\left\{{ }^{1} \mathbf{H}\right\}$ NMR spectrum of $[4](\mathrm{OTf})$ (in $\left.\mathrm{CD}_{3} \mathrm{CN}\right)$
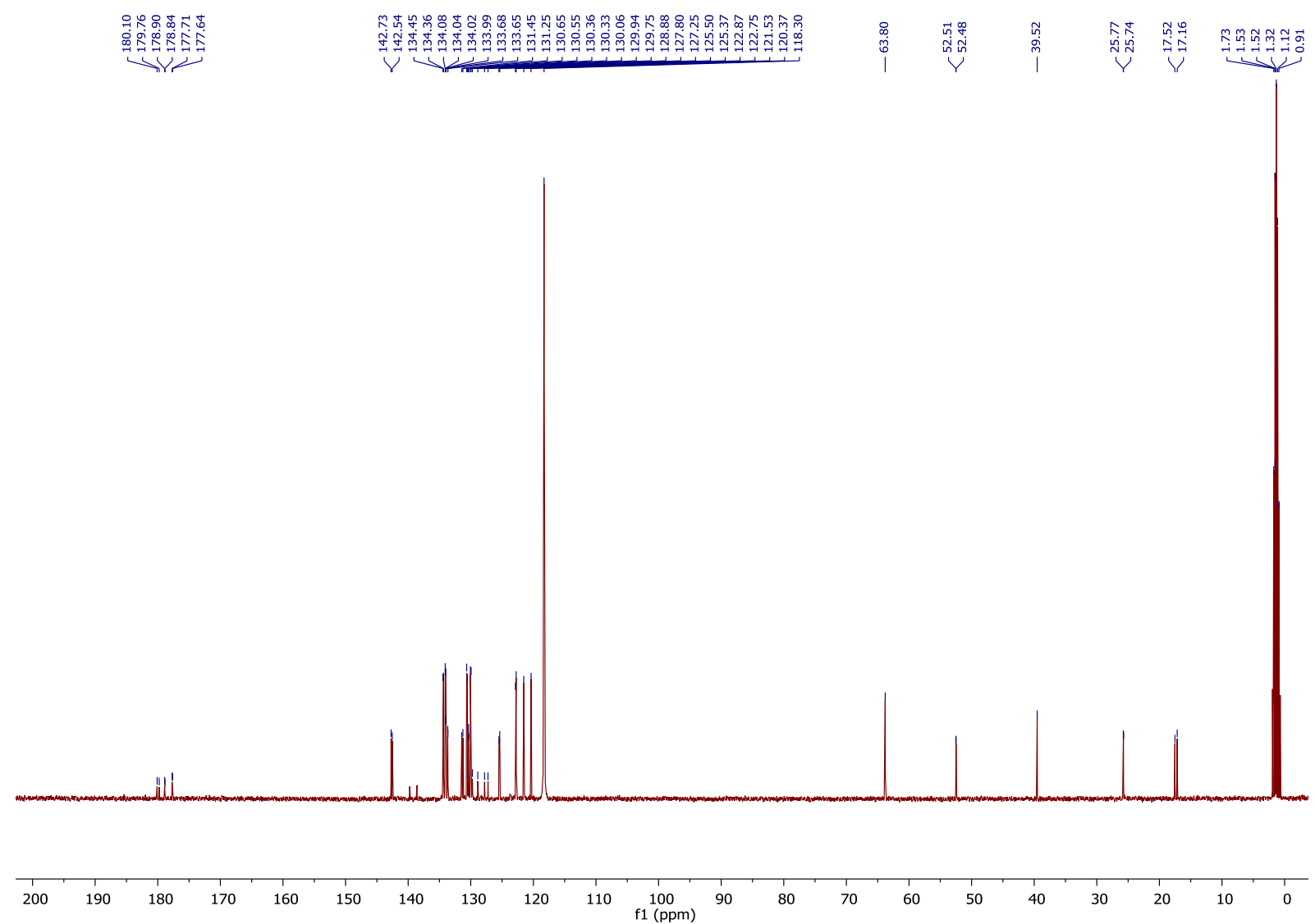

Fig. S10. ${ }^{31} \mathbf{P}\left\{{ }^{1} \mathbf{H}\right\}$ NMR spectrum of [5a](OTf $)_{2}\left(\right.$ in $\left.\mathrm{CD}_{3} \mathrm{CN}\right)$ 
Fig. S11. ${ }^{1} \mathbf{H}$ NMR spectrum of $[5 a](O T f)_{2}\left(\right.$ in $\left.\mathrm{CD}_{3} \mathrm{CN}\right)$

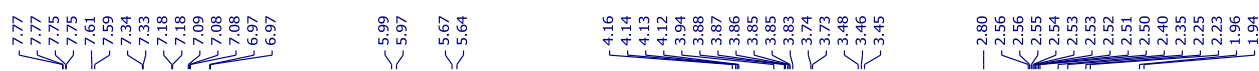

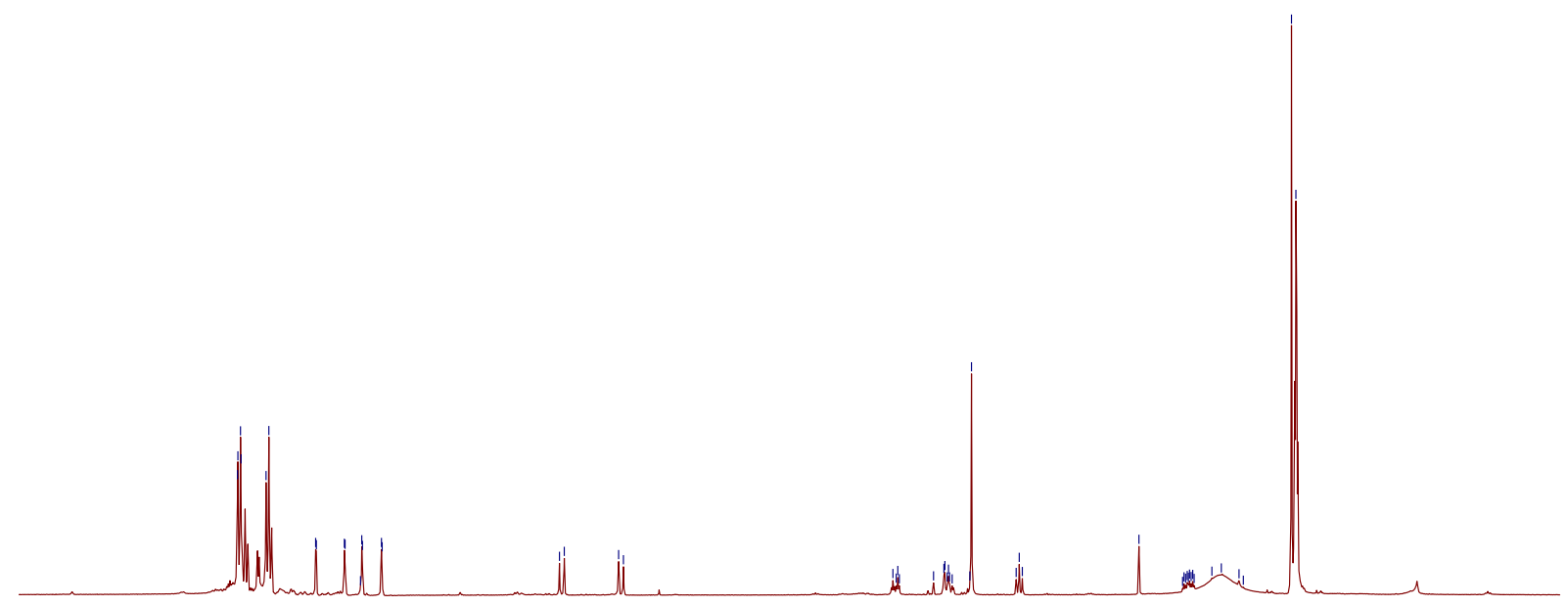

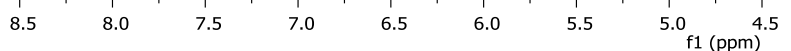

Fig. S12. ${ }^{13} \mathbf{C}\left\{{ }^{1} \mathbf{H}\right\}$ NMR spectrum of $[\mathbf{5 a}](\mathbf{O T f})_{2}\left(\right.$ in $\left.\mathrm{CD}_{3} \mathrm{CN}\right)$

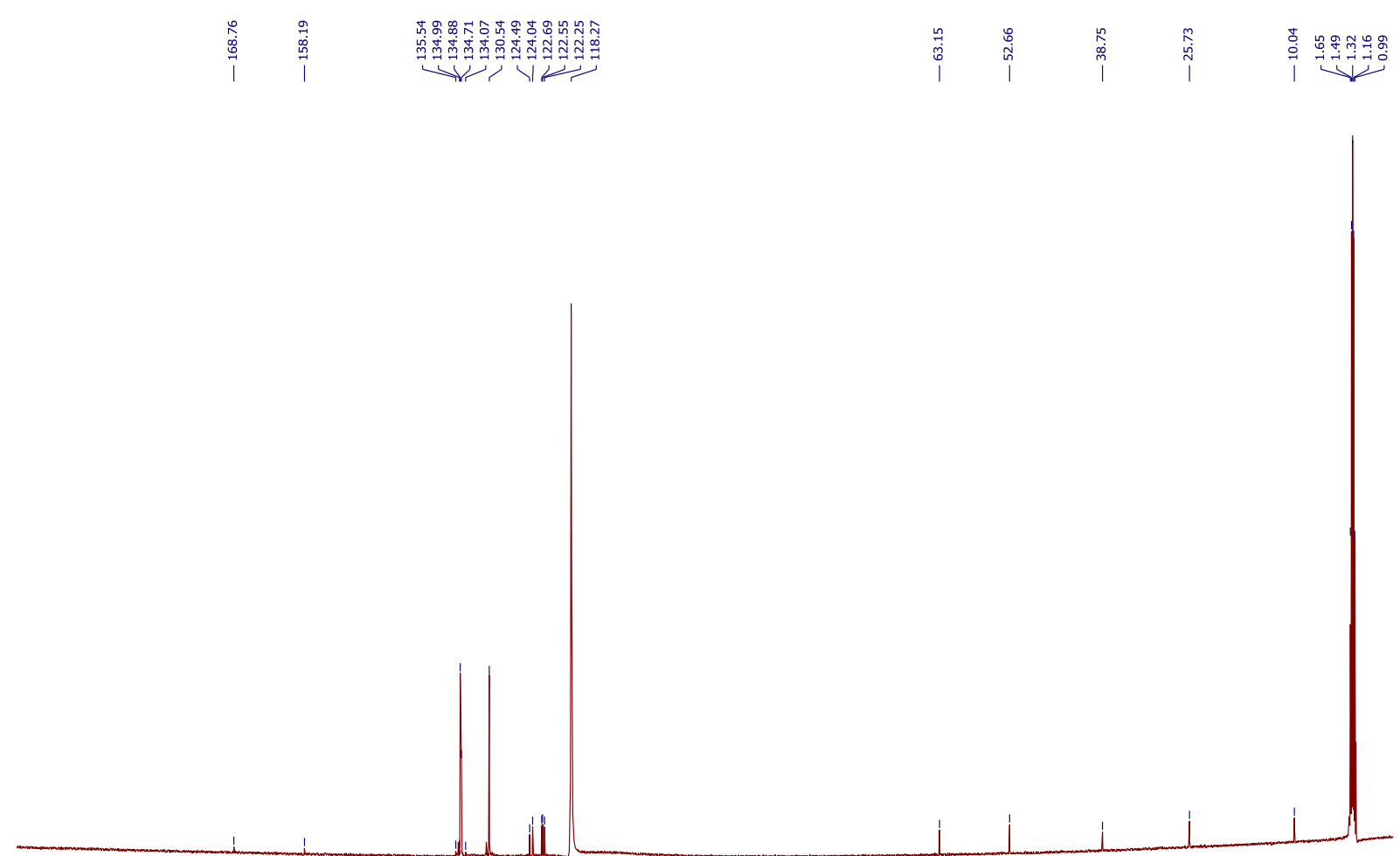


Fig. S13. ${ }^{31} \mathbf{P}\left\{{ }^{1} \mathbf{H}\right\}$ NMR spectrum of $[\mathbf{5 b}](\mathbf{O T f})_{2}\left(\right.$ in $\left.\mathrm{CD}_{2} \mathrm{Cl}_{2}\right)$

$\begin{array}{lllllllllllllllllllllllllllllllllll}100 & 95 & 90 & 85 & 80 & 75 & 70 & 65 & 60 & 55 & 50 & 45 & 40 & 35 & 30 & 25 & 20 & 15 & 10 & 5 & 0 & -5 & -10 & -15 & -20 & -25 & -30 & -35 & -40 & -45 & -5\end{array}$

Fig. S14. ${ }^{1} \mathbf{H}$ NMR spectrum of [5b](OTf) $)_{2}\left(\right.$ in $\left.\mathrm{CD}_{2} \mathrm{Cl}_{2}\right)$

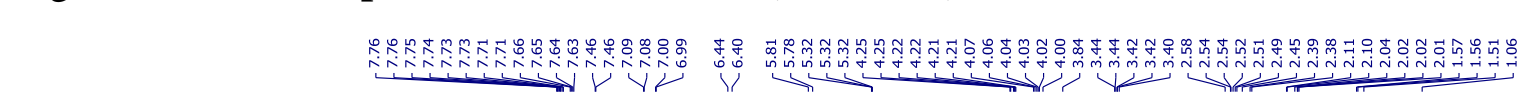

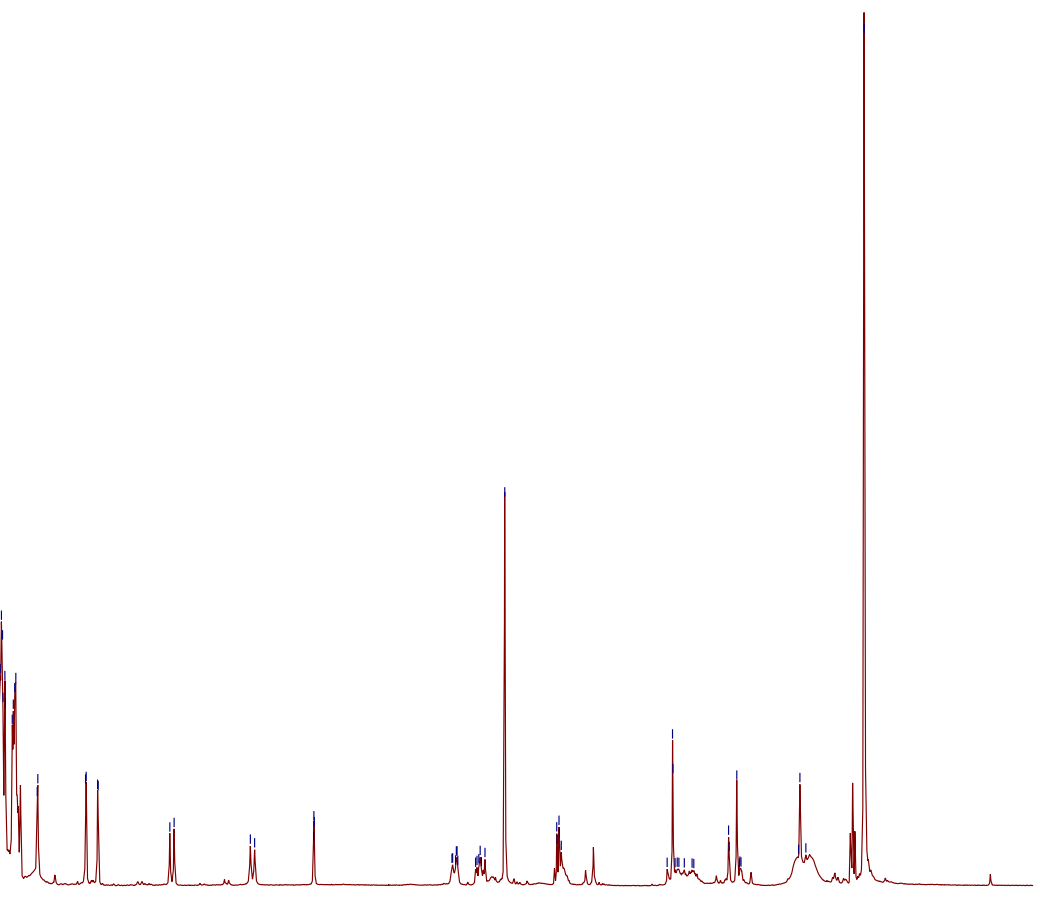

$\begin{array}{lllllllllllllllllllllllllll}11.5 & 11.0 & 10.5 & 10.0 & 9.5 & 9.0 & 8.5 & 8.0 & 7.5 & 7.0 & 6.5 & \begin{array}{c}6.0 \\ \mathrm{f} 1(\mathrm{ppm})\end{array} & 5.5 & 5.0 & 4.5 & 4.0 & 3.5 & 3.0 & 2.5 & 2.0 & 1.5 & 1.0 & 0.5 & 0.0\end{array}$ 
Fig. S15. ${ }^{13} \mathbf{C}\left\{{ }^{1} \mathbf{H}\right\}$ NMR spectrum of $[5 \mathbf{b}](\mathbf{O T f})_{2}\left(\right.$ in $\left.\mathrm{CD}_{2} \mathrm{Cl}_{2}\right)$

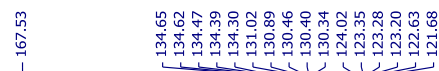

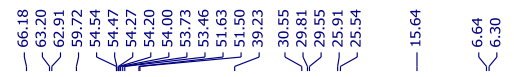
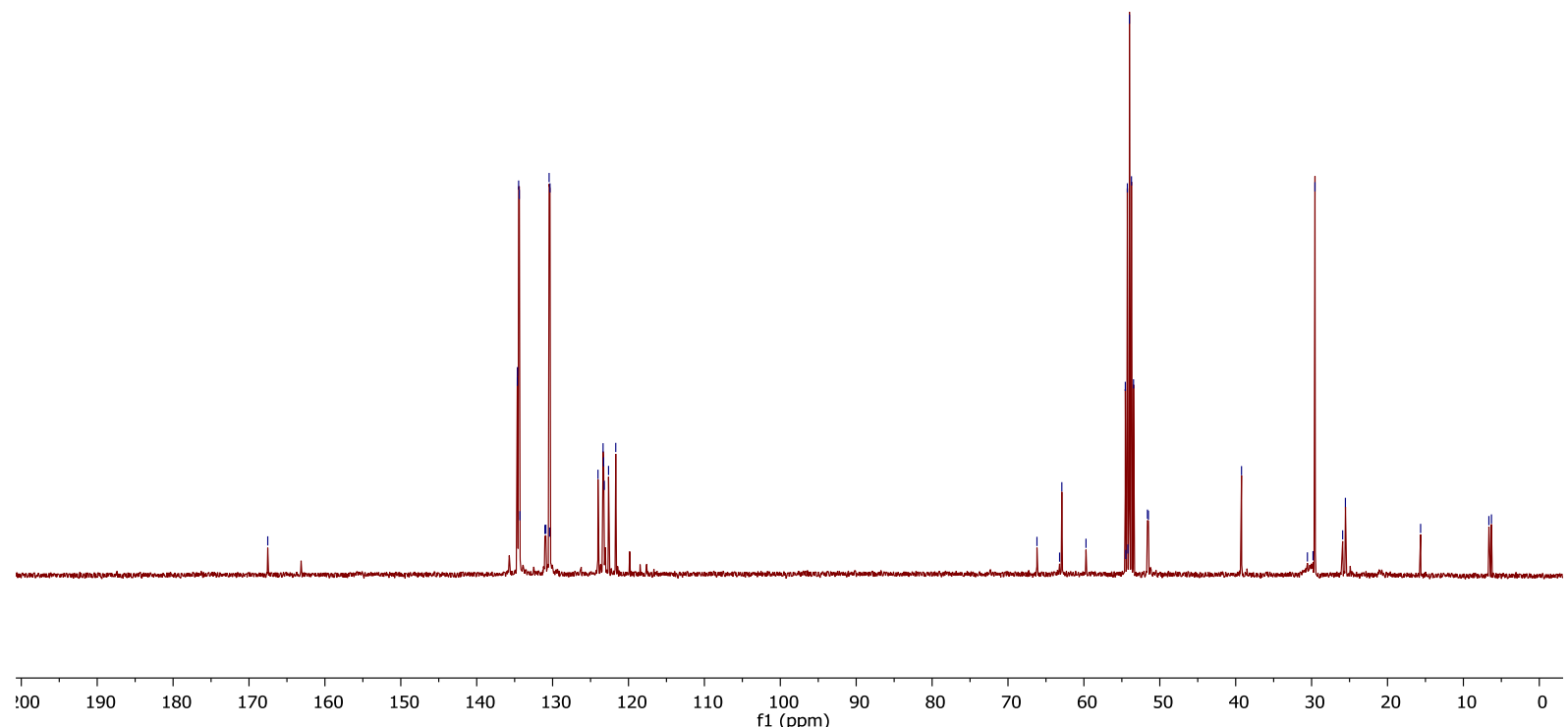

Fig. S16. IR of [5b](OTf $)_{2}\left(\right.$ in $\left.\mathrm{CH}_{2} \mathrm{Cl}_{2}\right)$

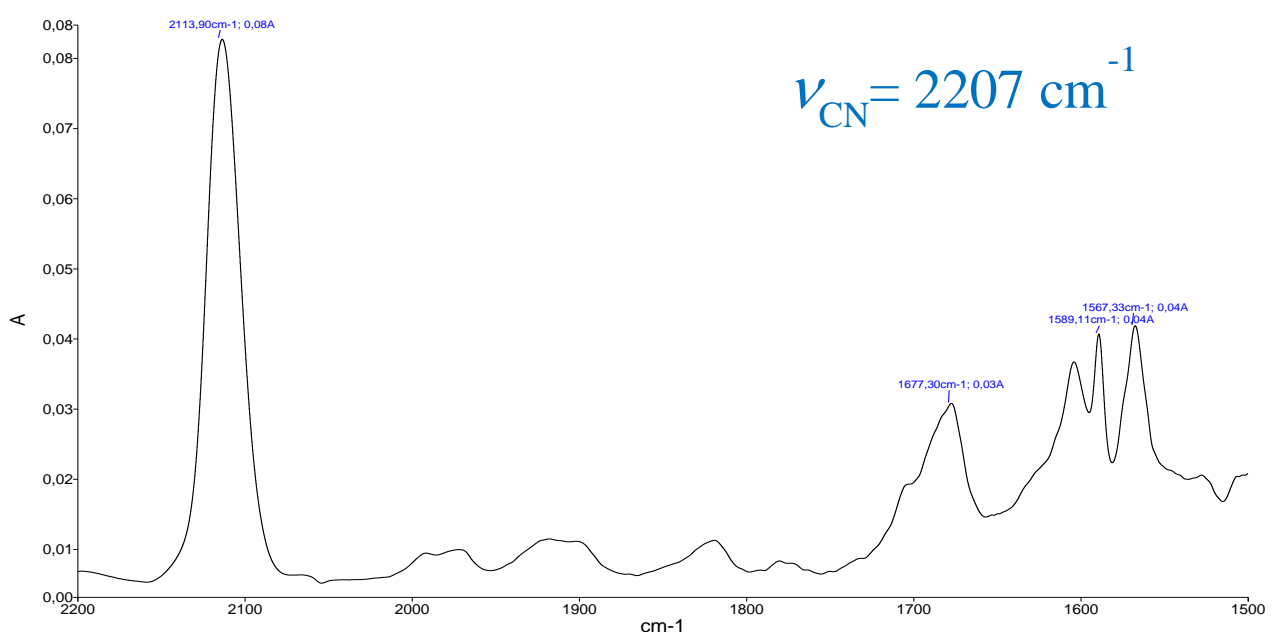


Fig. S17. ${ }^{31} \mathbf{P}\left\{{ }^{1} \mathbf{H}\right\}$ NMR spectrum of $[7] \mathrm{Br}_{2}\left(\right.$ in $\left.\mathrm{CDCl}_{3}\right)$

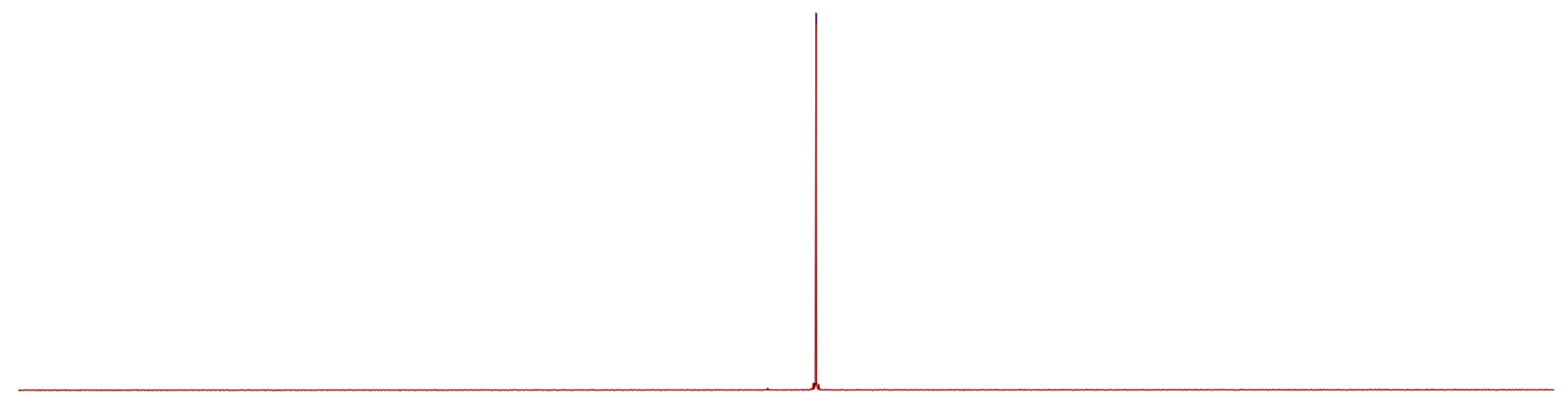

$\begin{array}{llllllllllllllllllllllllllllllll}100 & 95 & 90 & 85 & 80 & 75 & 70 & 65 & 60 & 55 & 50 & 45 & 40 & 35 & \begin{array}{l}30 \\ \mathrm{f} 1(\mathrm{ppm})\end{array} & 20 & 15 & 10 & 5 & 0 & -5 & -10 & -15 & -20 & -25 & -30 & -35 & -40 & -45 & -5\end{array}$

Fig. S18. ${ }^{1} \mathbf{H}$ NMR spectrum of [7]Br, $\left(\right.$ in $\left.\mathrm{CDCl}_{3}\right)$

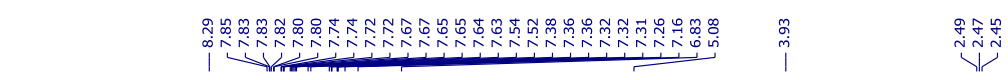

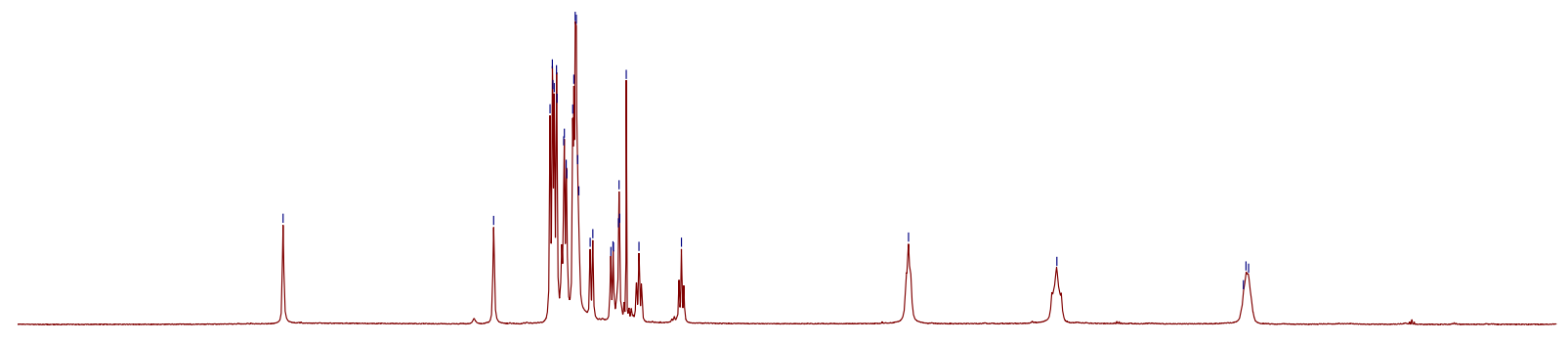

$\begin{array}{llllllllllllllllllllllll}11.5 & 11.0 & 10.5 & 10.0 & 9.5 & 9.0 & 8.5 & 8.0 & 7.5 & 7.0 & 6.5 & \begin{array}{c}6.0 \\ \mathrm{f} 1\end{array}(\mathrm{ppm}) & 5.5 & 5.0 & 4.5 & 4.0 & 3.5 & 3.0 & 2.5 & 2.0 & 1.5 & 1.0 & 0.5\end{array}$ 
Fig. S19. ${ }^{13} \mathbf{C}\left\{{ }^{1} \mathbf{H}\right\}$ NMR spectrum of $[7] \mathrm{Br}_{2}\left(\right.$ in $\left.\mathrm{CDCl}_{3}\right)$

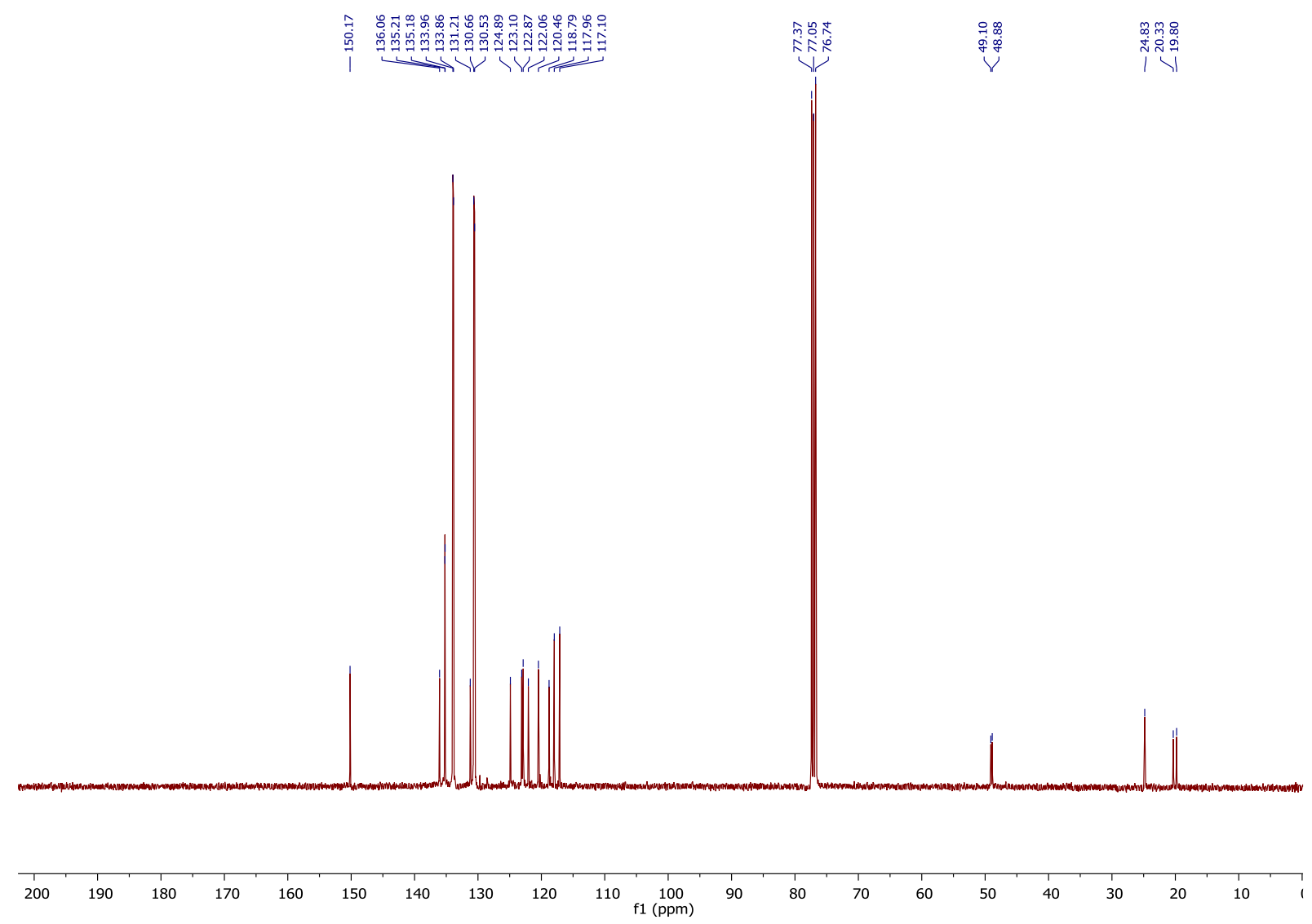

Fig. S20. ${ }^{31} \mathbf{P}\left\{{ }^{1} \mathbf{H}\right\}$ NMR spectrum of $[7](\mathbf{O T f})_{2}\left(\right.$ in $\left.\mathrm{CDCl}_{3}\right)$ 
Fig. S21. ${ }^{1} \mathbf{H}$ NMR spectrum of [7](OTf) $)_{2}$ (in $\mathrm{CDCl}_{3}$ )

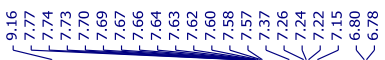

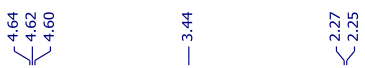

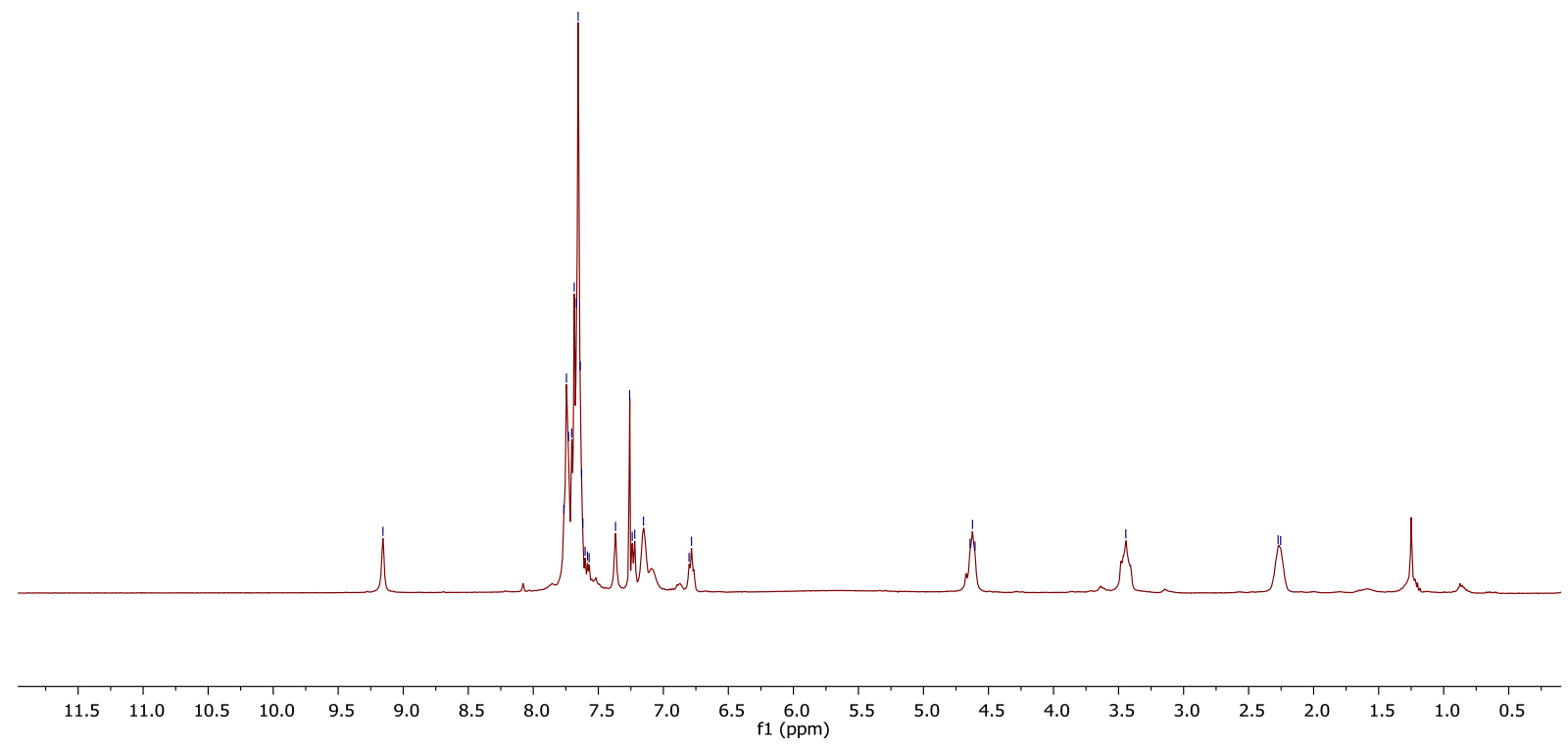

Fig. S22. ${ }^{13} \mathbf{C}\left\{{ }^{1} \mathbf{H}\right\}$ NMR spectrum of $[7](\mathbf{O T f})_{2}\left(\right.$ in $\left.\mathrm{CDCl}_{3}\right)$

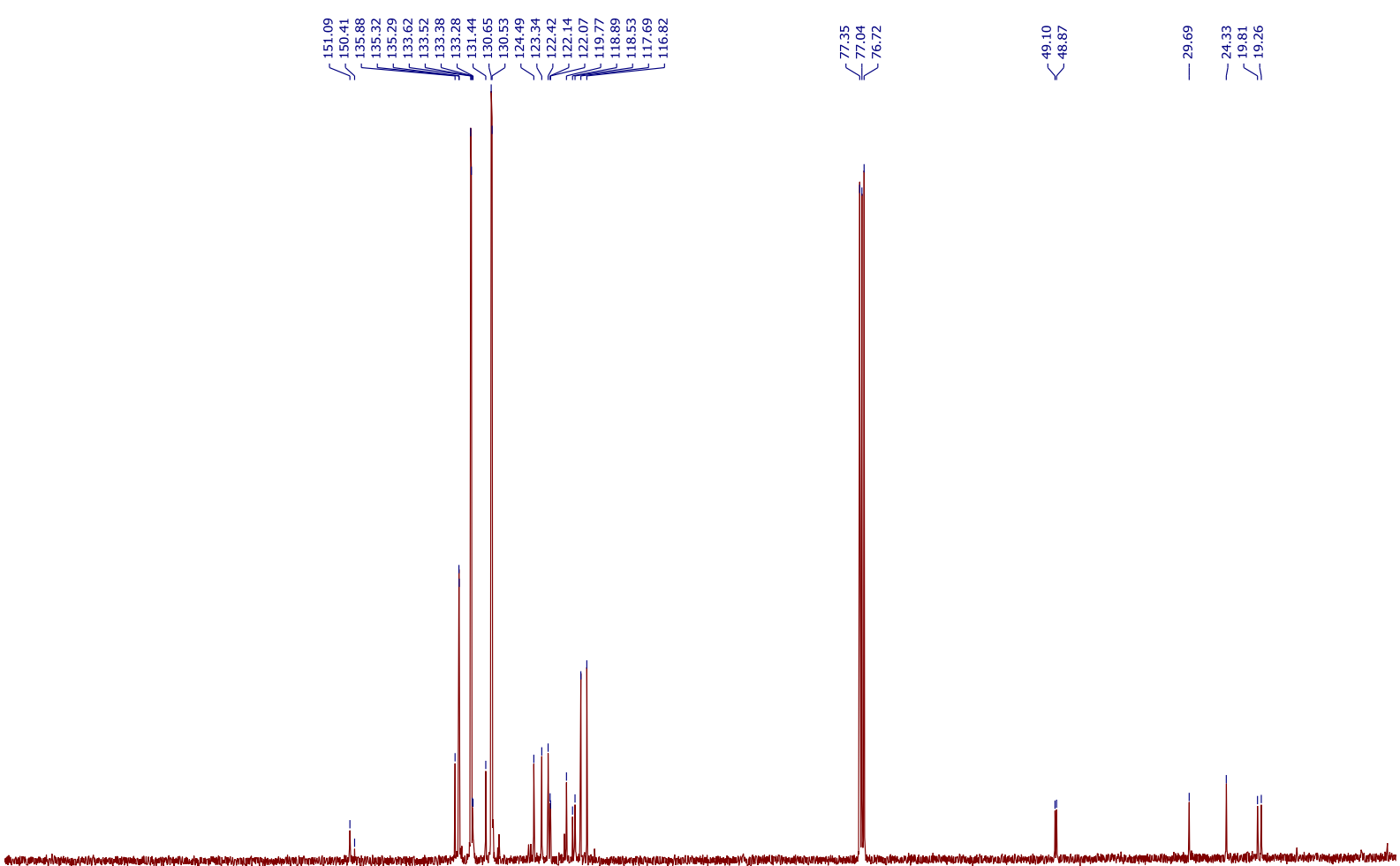


Fig. S23. ${ }^{31} \mathbf{P}\left\{{ }^{1} \mathbf{H}\right\}$ NMR spectrum of $[8](\mathbf{O T f})\left(\right.$ in $\left.\mathrm{CDCl}_{3}\right)$

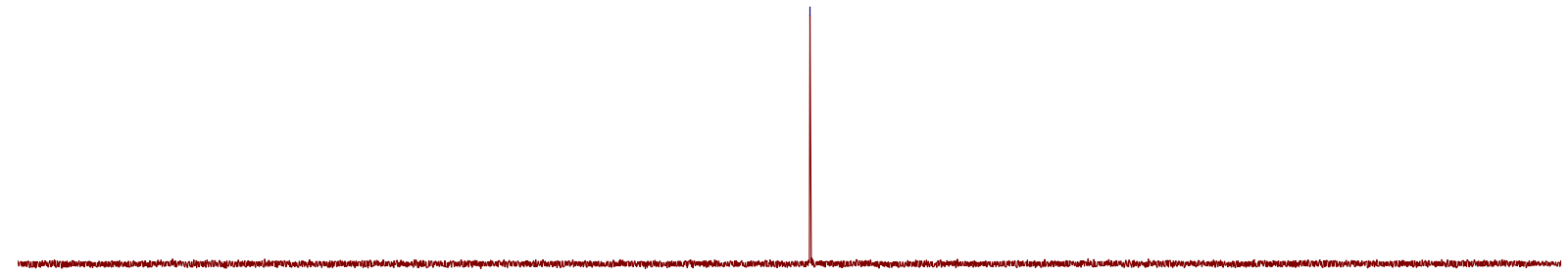

\begin{tabular}{rllllllllllllllllllllllllllllllllllllllllllll}
\hline 100 & 95 & 90 & 85 & 80 & 75 & 70 & 65 & 60 & 55 & 50 & 45 & 40 & 35 & 30 & 25 & 20 & 15 & 10 & 5 & 0 & -5 & -10 & -15 & -20 & -25 & -30 & -35 & -40 & -45 & -5
\end{tabular}

Fig. S24. ${ }^{1} \mathbf{H}$ NMR spectrum of $[8](\mathrm{OTf})\left(\right.$ in $\left.\mathrm{CDCl}_{3}\right)$

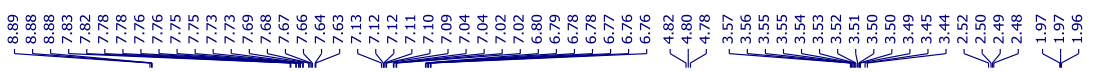

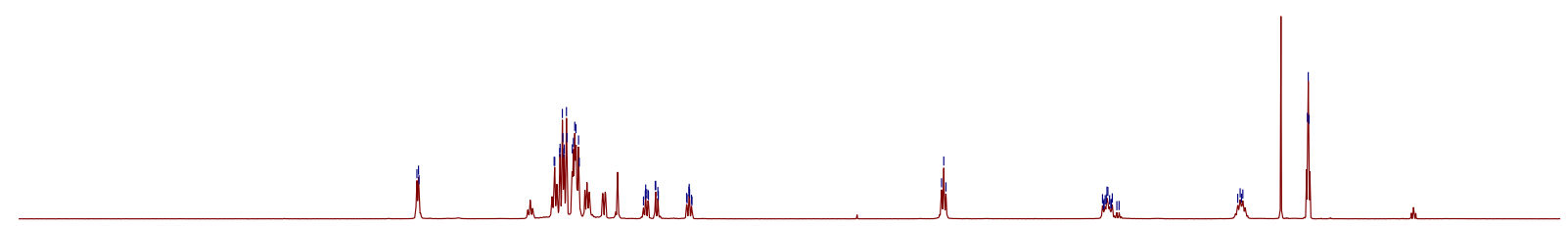

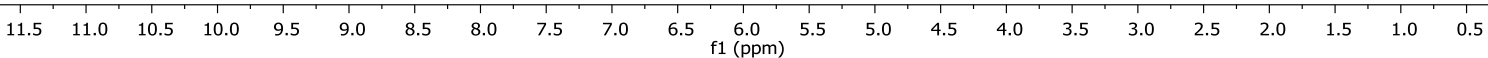


Fig. S25. ${ }^{13} \mathbf{C}\left\{{ }^{1} \mathbf{H}\right\}$ NMR spectrum of $[8](\mathrm{OTf})\left(\right.$ in $\left.\mathrm{CDCl}_{3}\right)$
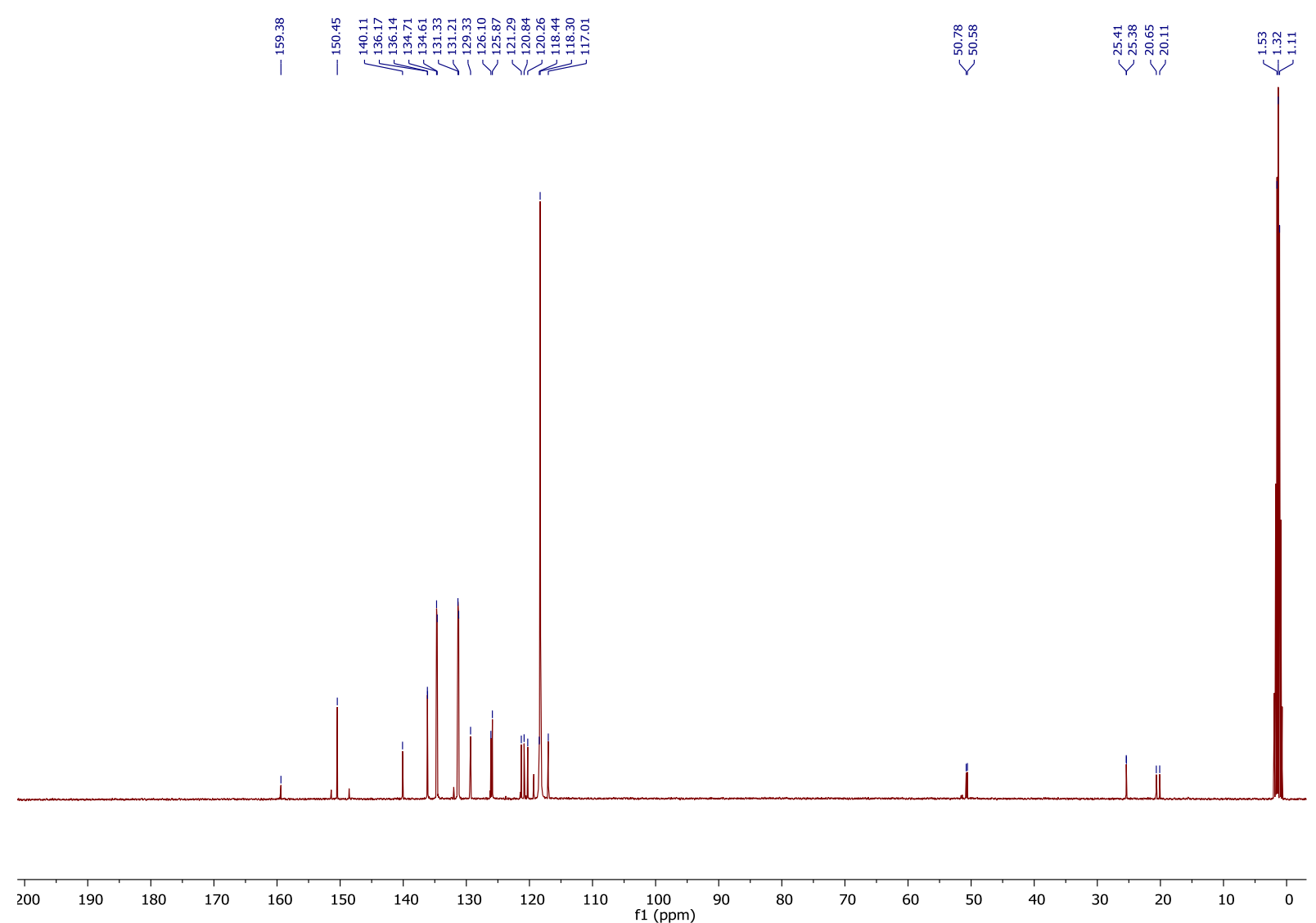

Fig. S26. ${ }^{31} \mathbf{P}\left\{{ }^{1} \mathbf{H}\right\}$ NMR spectrum of $[9](\mathbf{O T f})_{2}\left(\right.$ in $\left.\mathrm{CD}_{3} \mathrm{CN}\right)$ 
Fig. S27. ${ }^{1} \mathrm{H}$ NMR spectrum of [9](OTf $)_{2}\left(\right.$ in $\left.\mathrm{CD}_{3} \mathrm{CN}\right)$

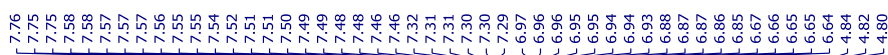

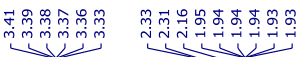

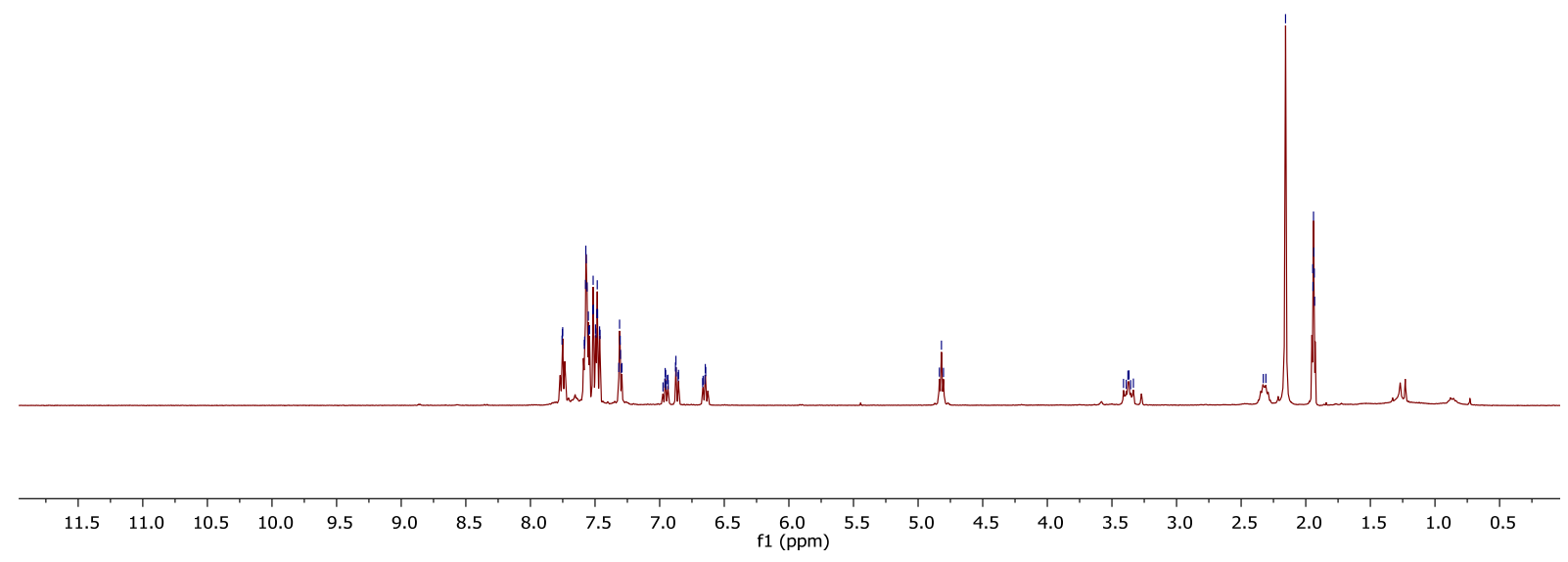

Fig. S28. ${ }^{13} \mathbf{C}\left\{{ }^{1} \mathbf{H}\right\}$ NMR spectrum of $[9](\text { OTf })_{2}\left(\right.$ in $\left.\mathrm{CD}_{3} \mathrm{CN}\right)$
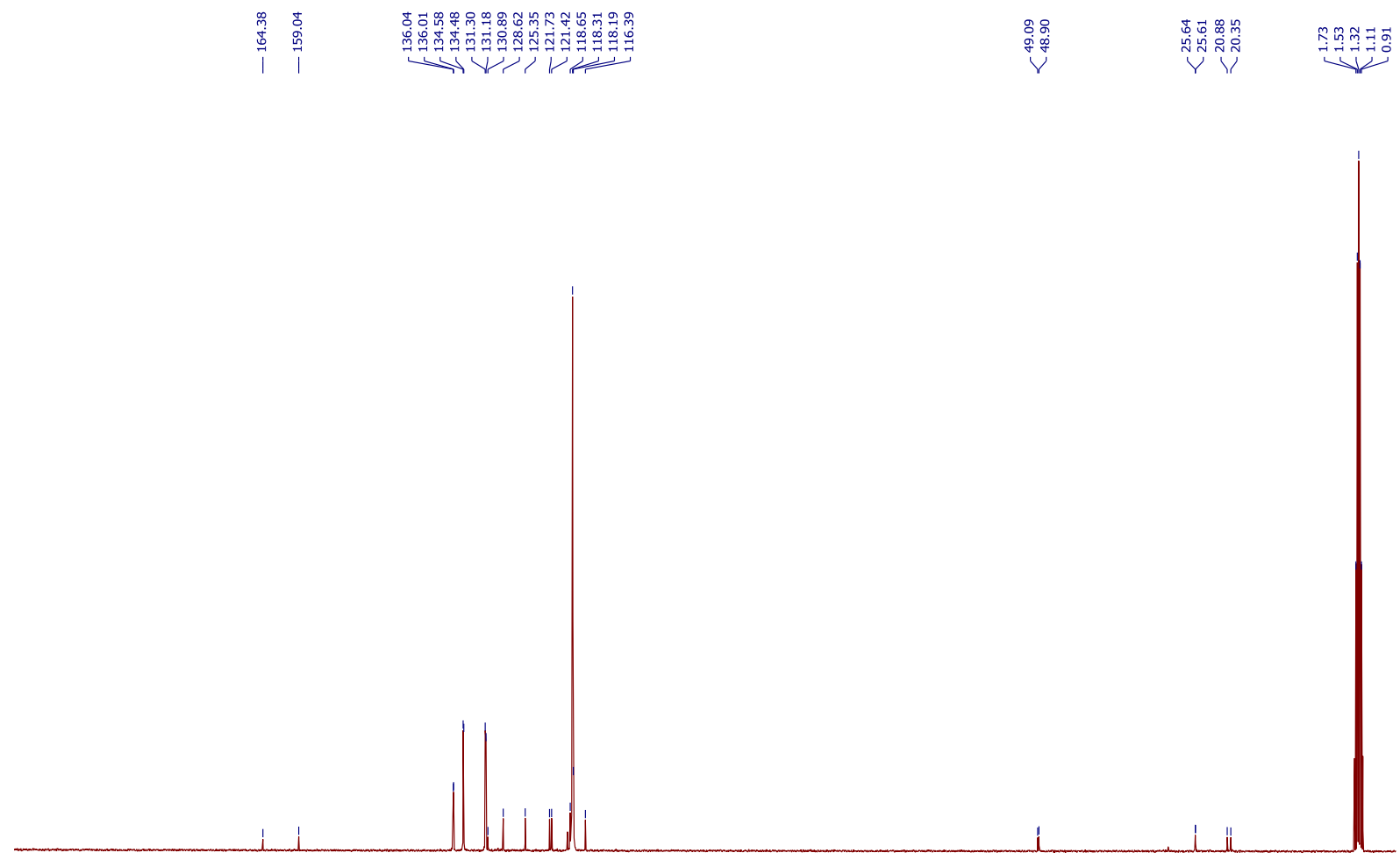

200

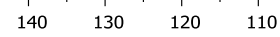

100

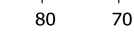


Fig. S29. ${ }^{31} \mathbf{P}\left\{{ }^{1} \mathbf{H}\right\}$ NMR spectrum of $\mathbf{1 0}\left(\right.$ in $\left.\mathrm{CD}_{2} \mathrm{Cl}_{2}\right)$

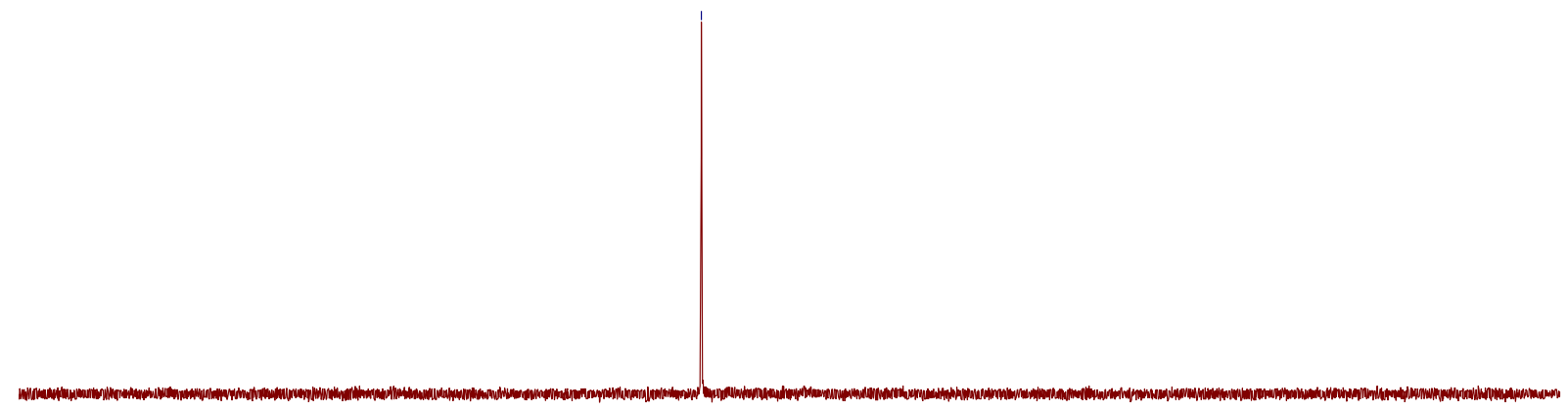
\begin{tabular}{llllllllllllllllllllllllllllllllllllllllllll}
\hline 100 & 95 & 90 & 85 & 80 & 75 & 70 & 65 & 60 & 55 & 50 & 45 & 40 & 35 & 30 & 25 & 20 & 15 & 10 & 5 & 0 & -5 & -10 & -15 & -20 & -25 & -30 & -35 & -40 & -45 & -56
\end{tabular}

Fig. S30. ${ }^{1} \mathbf{H}$ NMR spectrum of $\mathbf{1 0}$ (in $\mathrm{CD}_{2} \mathrm{Cl}_{2}$ )

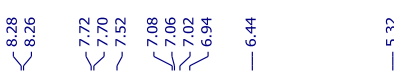

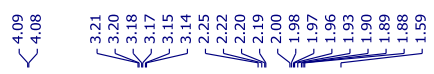

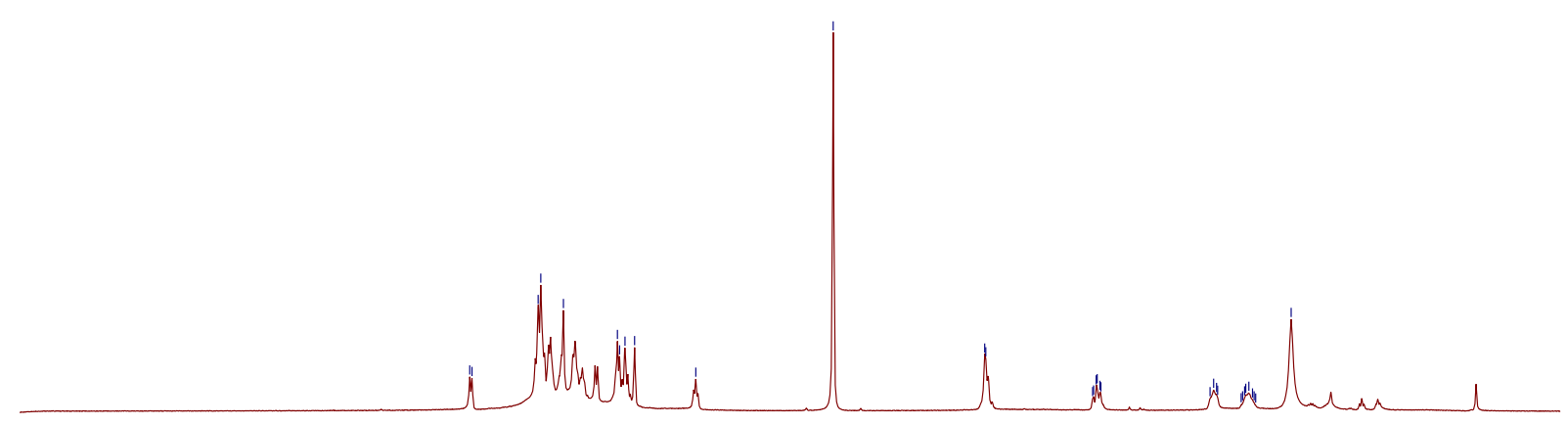

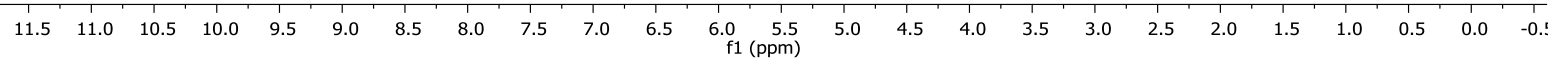


Fig. S31. ${ }^{13} \mathbf{C}\left\{{ }^{1} \mathbf{H}\right\}$ NMR spectrum of 10 (in $\mathrm{CD}_{3} \mathrm{CN}$ )

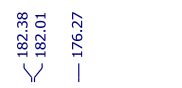

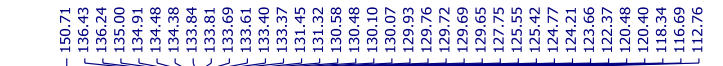

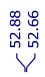

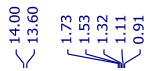
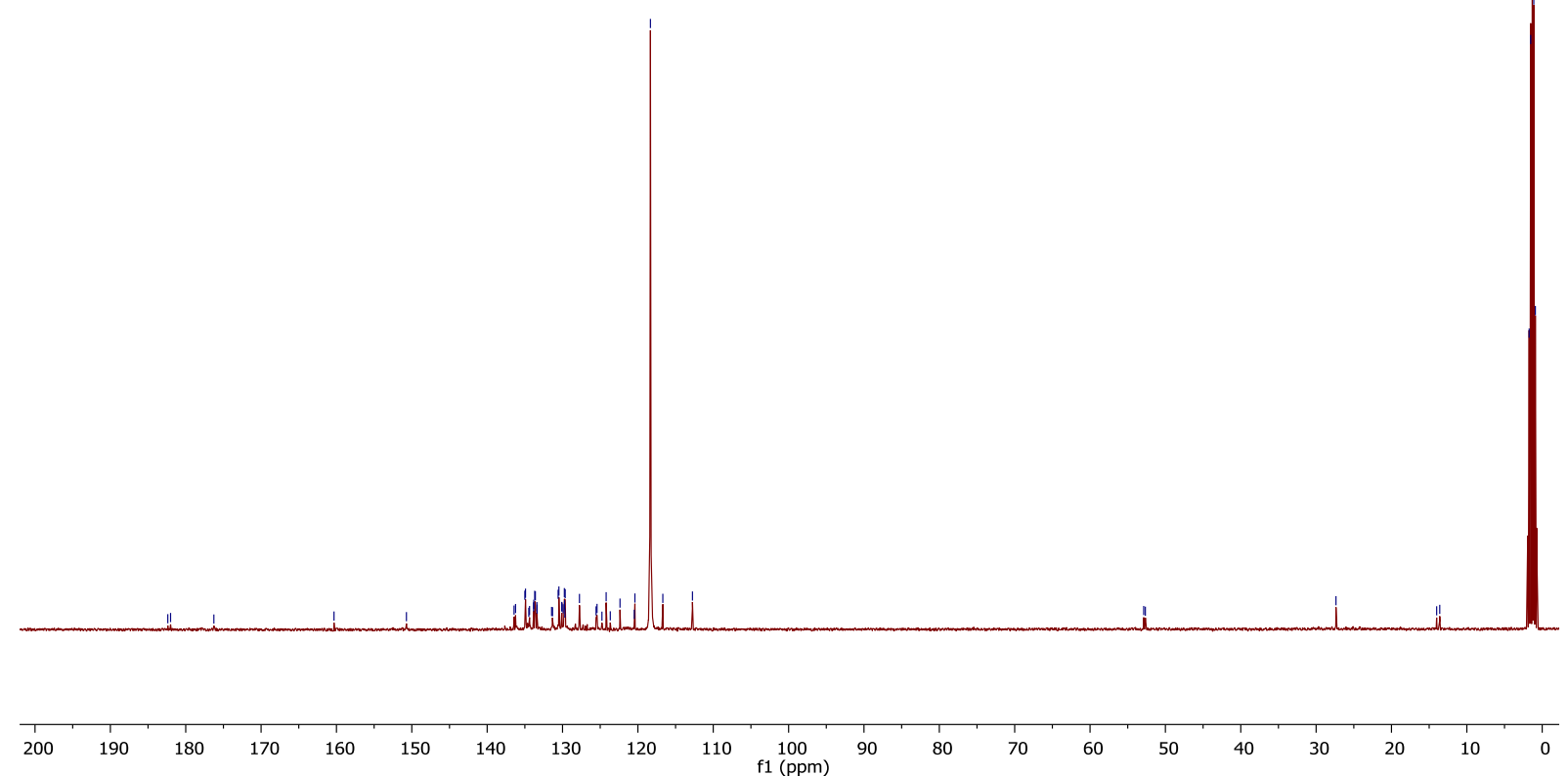

Fig. S32. ${ }^{31} \mathbf{P}\left\{{ }^{1} \mathbf{H}\right\}$ NMR spectrum of $[11 a](\mathrm{OTf})\left(\right.$ in $\left.\mathrm{CD}_{3} \mathrm{CN}\right)$

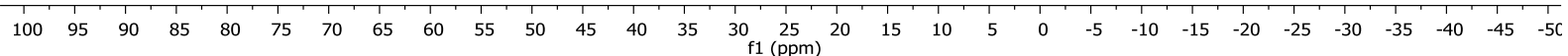


Fig. S33. ${ }^{1} \mathrm{H}$ NMR spectrum of [11a](OTf) (in $\left.\mathrm{CD}_{3} \mathrm{CN}\right)$

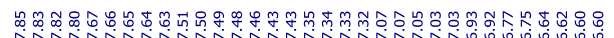

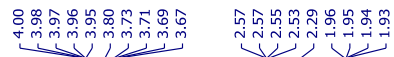

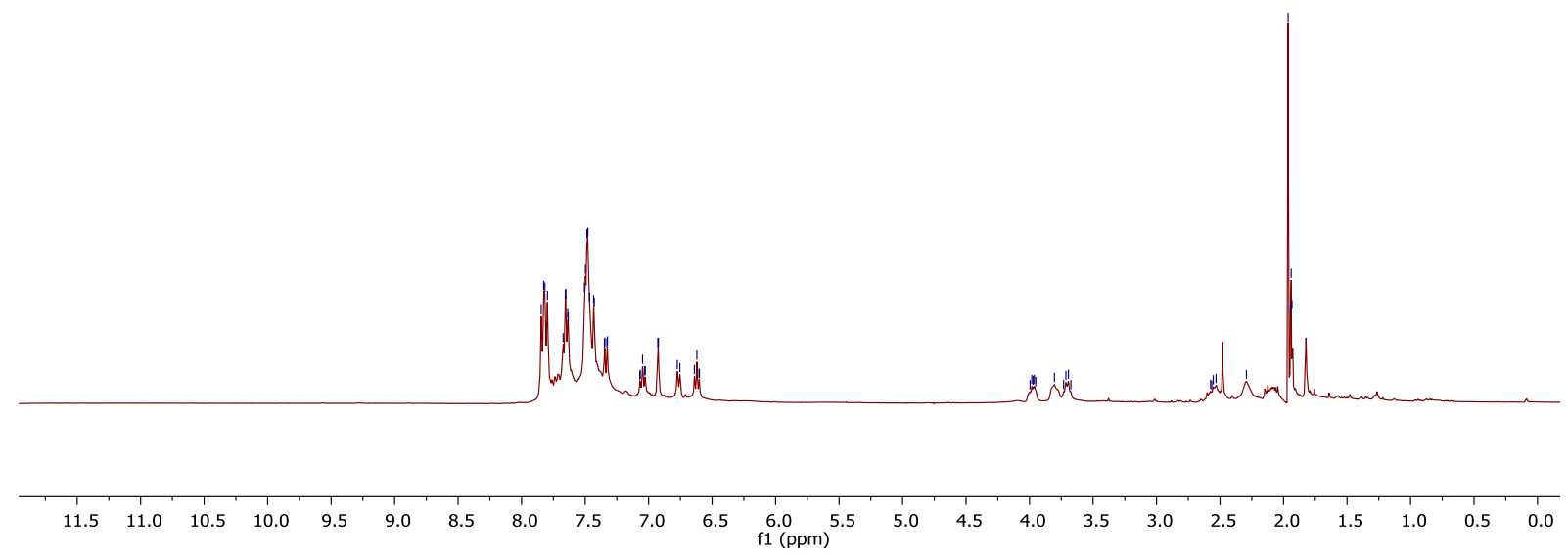

Fig. S34. ${ }^{13} \mathbf{C}\left\{{ }^{1} \mathbf{H}\right\}$ NMR spectrum of $[11 \mathrm{a}](\mathrm{OTf})\left(\right.$ in $\left.\mathrm{CD}_{3} \mathrm{CN}\right)$
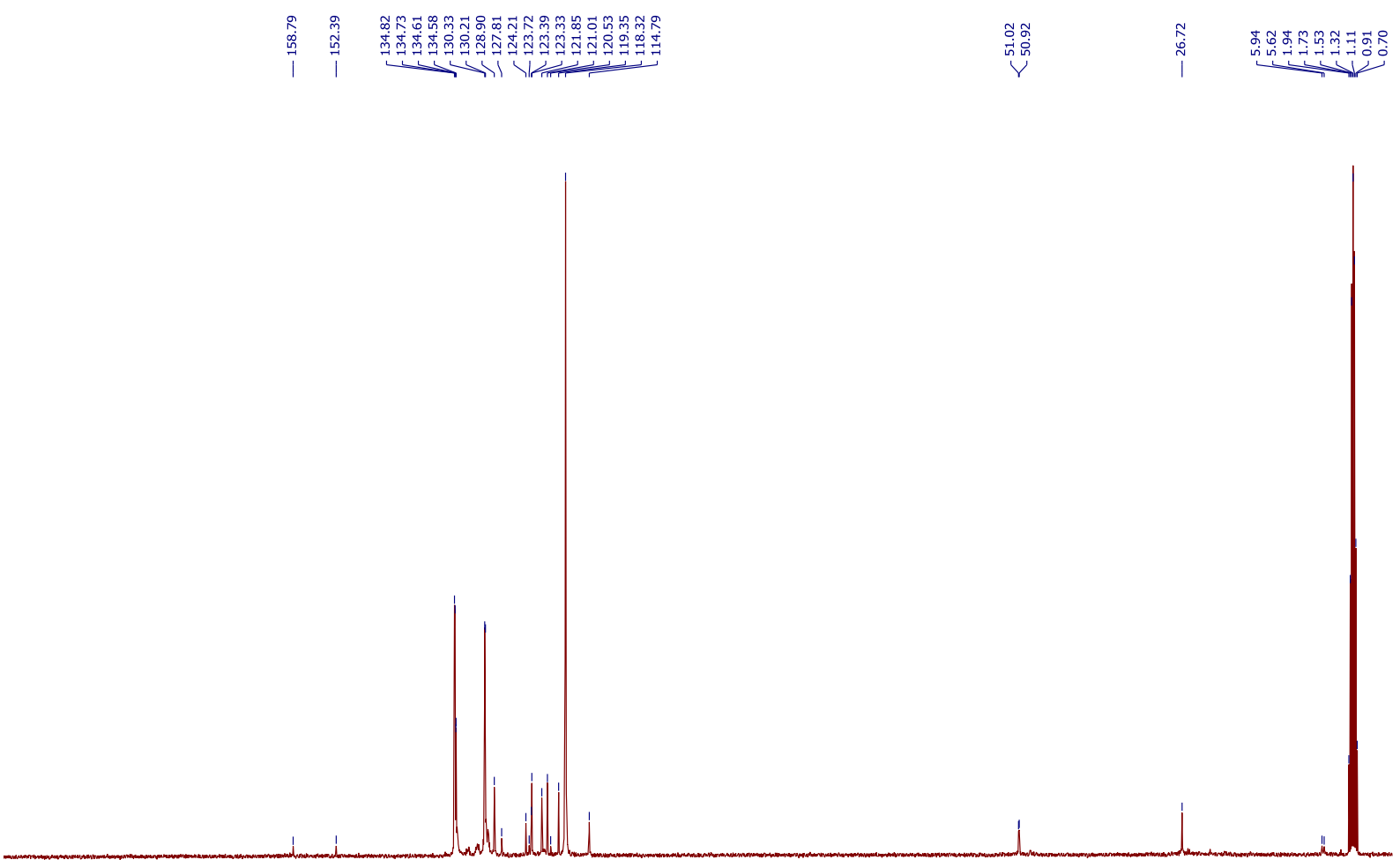

200

19 $140 \quad 130 \quad 120$ 110 100 80 
Fig. S37. ${ }^{13} \mathbf{C}\left\{{ }^{1} \mathbf{H}\right\}$ NMR spectrum of $[\mathbf{1 1 b}](\mathbf{O T f})\left(\right.$ in $\left.\mathrm{CD}_{2} \mathrm{Cl}_{2}\right)$
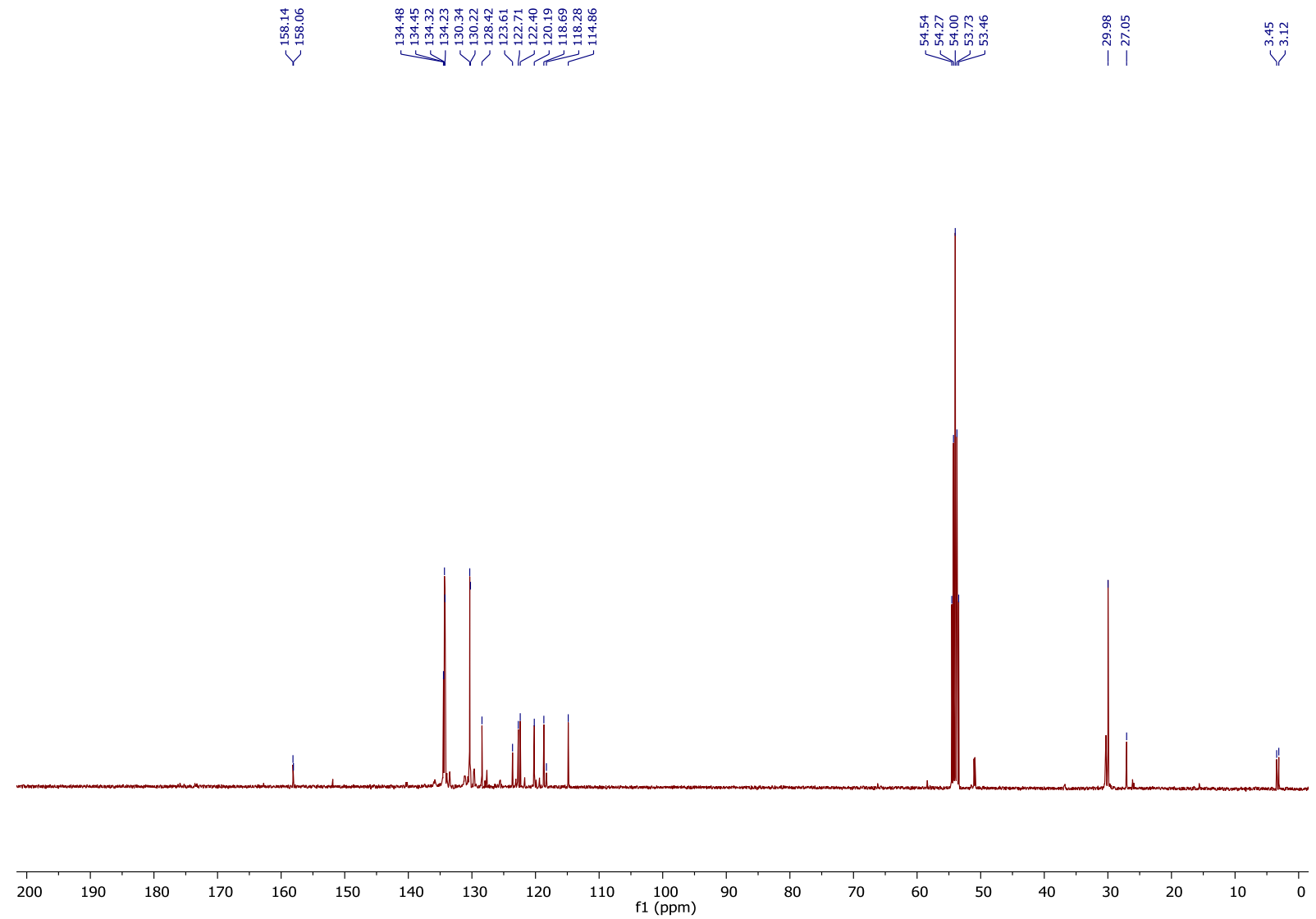

Fig. S38. IR of [11b](OTf) in $\mathrm{CH}_{2} \mathrm{Cl}_{2}$

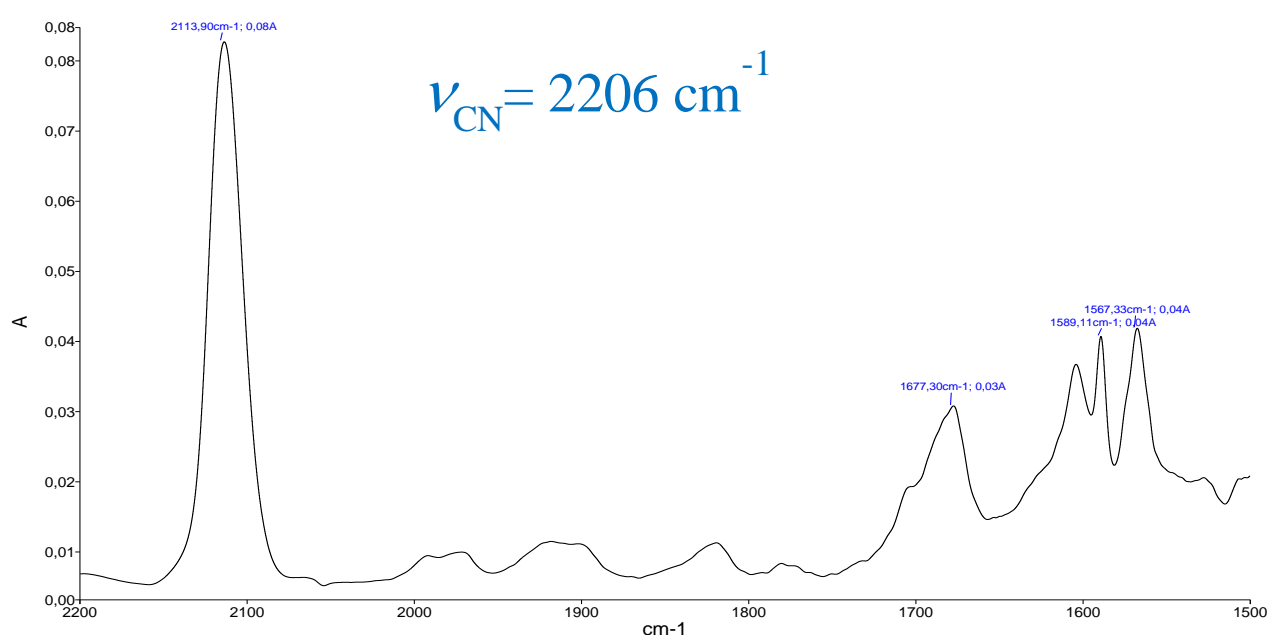


Table S1. Crystallographic table for [4](OTf), [7]Br2, [8](OTf), [9](OTf) 2 , and 10

\begin{tabular}{|c|c|c|c|c|c|}
\hline & {$[4](\mathrm{OTf})$} & {$[7] \mathrm{Br}_{2}$} & {$[8](\mathbf{O T f})$} & {$[9](\text { OTf })_{2}$} & 10 \\
\hline $\begin{array}{l}\text { Empirical } \\
\text { formula }\end{array}$ & $\begin{array}{l}\mathrm{C}_{31} \mathrm{H}_{30} \mathrm{Cl}_{2} \mathrm{~F}_{3} \mathrm{~N}_{4} \\
\mathrm{O}_{3} \mathrm{PPdS}\end{array}$ & $\mathrm{C}_{30} \mathrm{H}_{29} \mathrm{Br}_{2} \mathrm{~N}_{2} \mathrm{OP}$ & $\begin{array}{l}\mathrm{C}_{37.5} \mathrm{H}_{33.5} \mathrm{Cl}_{5.5} \mathrm{~F}_{3} \\
\mathrm{~N}_{3} \mathrm{O}_{4} \mathrm{PPdS}\end{array}$ & $\begin{array}{l}\mathrm{C}_{66} \mathrm{H}_{66} \mathrm{~F}_{6} \mathrm{~N}_{4} \mathrm{O}_{10} \mathrm{P}_{2} \\
\mathrm{PdS}_{2}\end{array}$ & $\mathrm{C}_{30} \mathrm{H}_{25} \mathrm{~N} 2 \mathrm{OPPd}$ \\
\hline $\begin{array}{l}\text { Formula } \\
\text { Mass }\end{array}$ & 803.94 & 624.32 & 1011.62 & 1421.72 & 566.91 \\
\hline Crystal system & Monoclinic & Triclinic & Monoclinic & Triclinic & Monoclinic \\
\hline Space group & $\mathrm{P} 2{ }_{1} / \mathrm{c}$ & $\mathrm{P}-1$ & $\mathrm{P} 2{ }_{1} / \mathrm{c}$ & $\mathrm{P}-1$ & $\mathrm{P} 2{ }_{1} / \mathrm{c}$ \\
\hline $\mathrm{T}[\mathrm{K}]$ & 100 & 100 & 107 & 110 & 100 \\
\hline$a[\AA]$ & $11.6634(8)$ & $8.5885(6)$ & 19.3843(7) & $9.3094(14)$ & $9.84220(2)$ \\
\hline$b[\AA]$ & $15.5910(10)$ & 11.6377(8) & $12.5986(4)$ & $13.2185(18)$ & $20.88700(3)$ \\
\hline$c[\AA]$ & $18.0431(13)$ & $14.1355(10)$ & 19.0953(6) & $14.367(2)$ & $11.87830(2)$ \\
\hline$\square\left[{ }^{\circ}\right]$ & 90 & $103.085(2)$ & 90 & $114.718(4)$ & 90 \\
\hline$\square\left[{ }^{\circ}\right]$ & $101.141(2)$ & $100.336(3)$ & $118.9705(11)$ & $91.308(4)$ & $107.837(2)$ \\
\hline$\square\left[^{\circ}\right]$ & 90 & $92.704(2)$ & 90 & $97.971(4)$ & 90 \\
\hline$V\left[\AA^{3}\right]$ & $3219.2(4)$ & $1348.1(2)$ & $4079.8(2)$ & $1584.0(4)$ & $2324.50(3)$ \\
\hline$D_{c}$ & 1.659 & 1.538 & 1.647 & 1.488 & 1.620 \\
\hline$Z$ & 4 & 2 & 4 & 1 & 4 \\
\hline$\mu\left[\mathrm{mm}^{-1}\right]$ & 0.915 & 3.092 & 0.963 & 0.490 & 0.895 \\
\hline$\lambda(\AA)$ & 0.71073 & 0.71073 & 0.71073 & 0.71073 & 0.71073 \\
\hline Refl. measured & 57176 & 66061 & 90363 & 35771 & 144026 \\
\hline Refl. unique/ $R_{i \mathrm{n} t}$ & $6103 / 0.11$ & $5930 / 0.04$ & $10121 / 0.04$ & $5783 / 0.15$ & $7085 / 0.05$ \\
\hline $\begin{array}{l}\text { Refl. with } I> \\
n \square(I)\end{array}$ & $4168, n=2.3$ & $5409, \mathrm{n}=3$ & $8562, n=3$ & $3525, \mathrm{n}=2.4$ & $6400, n=3$ \\
\hline $\mathrm{Nb}$ parameters & 415 & 325 & 514 & 436 & 316 \\
\hline Refinement on & $\mathrm{F}$ & $\mathrm{F}$ & $\mathrm{F}$ & $\mathrm{F}$ & $\mathrm{F}$ \\
\hline$R$ with $I>n \square(I)$ & 0.0448 & 0.0232 & 0.0454 & 0.0557 & 0.0207 \\
\hline$R_{w}$ with $I>n \square(I)$ & 0.0447 & 0.0239 & 0.0479 & 0.0566 & 0.0211 \\
\hline GooF & 1.112 & 1.043 & 1.028 & 1.134 & 0.829 \\
\hline $\begin{array}{l}\square \square \max / \square \square \min \\
{\left[\mathrm{e} . \AA^{-3}\right]}\end{array}$ & $1.57 /-0.72$ & $0.88 /-0.36$ & $1.19 /-1.86$ & $1.06 /-1.01$ & $0.53 /-0.49$ \\
\hline
\end{tabular}


Fig. S39. Optimized geometries of Pd complex $5^{2+}$ at the PBE-D3/6-31G**/LANL2DZ* (Pd) level of calculation: $\mathbf{L}=\mathbf{C N} t \mathbf{B u}(l e f t)$ and $\mathbf{L}=\mathbf{C O}$ (right)
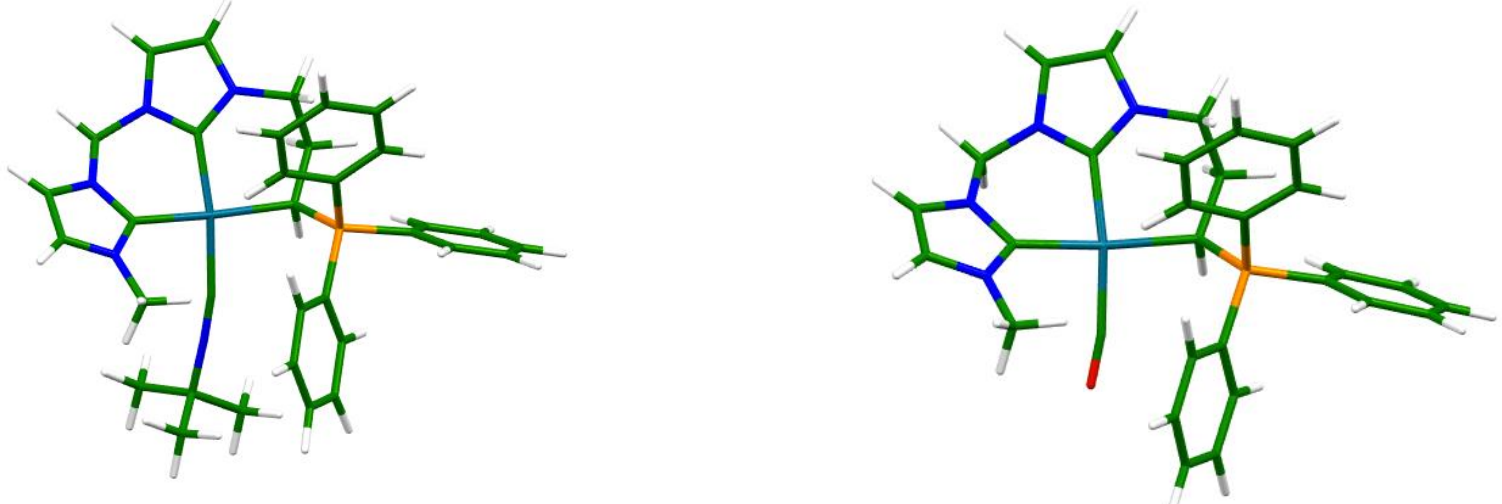

Fig. S40. Optimized geometry of Pd complex $11^{+}$at the PBE-D3/6-31G**/LANL2DZ* (Pd) level of calculation: $\mathbf{L}=\mathbf{C N} t \mathbf{B u}$ (left) and $\mathbf{L}=\mathbf{C O}$ (right)
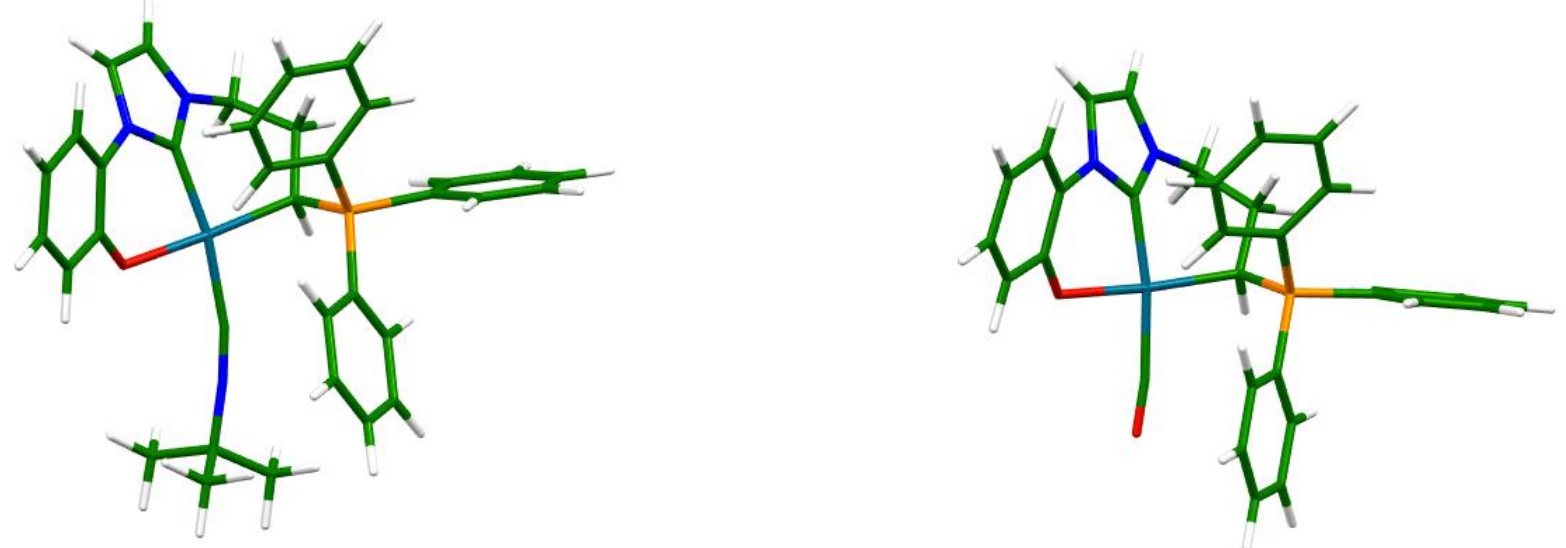
Fig. S41. Optimized geometry of Pd complex $12^{2+}$ at the PBE-D3/6-31G**/LANL2DZ* (Pd) level of calculation: $\mathbf{L}=\mathbf{C N} t \mathbf{B u}$ (left) and $\mathbf{L}=\mathbf{C O}$ (right). In both cases, only the $d l$ isomer is represented.
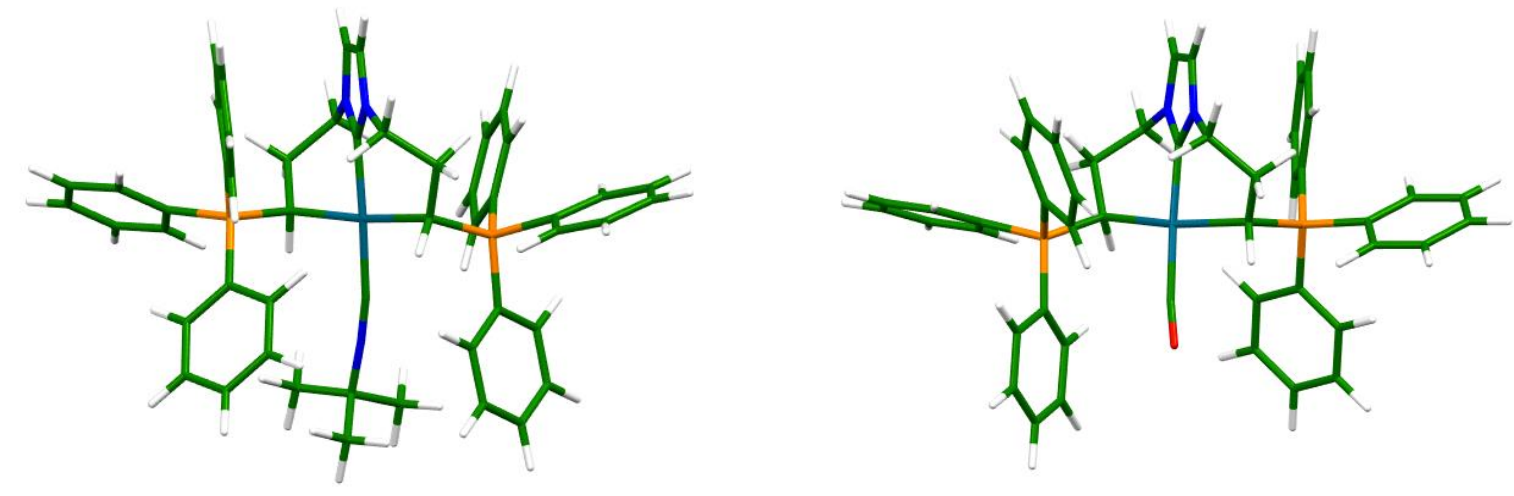

Fig. S42. Optimized geometry of Pd complex $13^{+}$at the PBE-D3/6-31G**/LANL2DZ* (Pd) level of calculation: only the $d l$-isomer is represented.

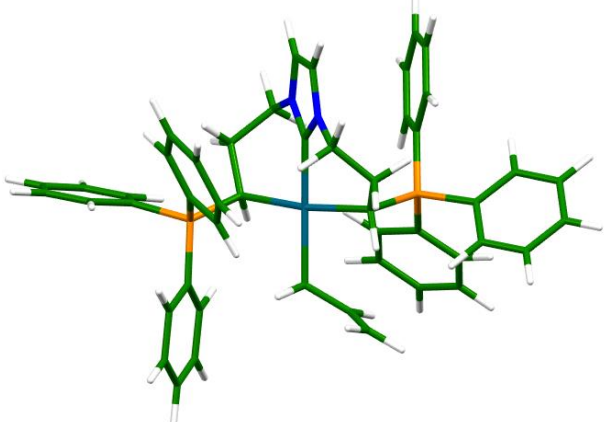

